\title{
Mechanism of glycogen synthase inactivation and interaction with
} glycogenin

Laura Marr ${ }^{a}$, Dipsikha Biswas ${ }^{b}$, Leonard A. Daly ${ }^{c}$, Christopher Browning ${ }^{d}$, John Pollard ${ }^{d}$, Catherine Hudson $^{d}$, Jay Bertrand ${ }^{d}$, Neil A. Ranson ${ }^{a}$, Heena Khatter ${ }^{d}$, Claire E. Eyers ${ }^{c}$, Kei

a Astbury Centre for Structural Molecular Biology, School of Molecular and Cellular Biology, Faculty of Biological Sciences, University of Leeds, Leeds, UK

${ }^{b}$ Novo Nordisk Foundation Center for Basic Metabolic Research, University of Copenhagen, Copenhagen, Denmark

${ }^{c}$ Department of Biochemistry and Systems Biology, Institute of Systems, Molecular and Integrative Biology, University of Liverpool, Liverpool, UK ${ }^{d}$ Vertex Pharmaceuticals Inc., Milton Park, Abingdon, UK

*Correspondence to: e.zeqiraj@leeds.ac.uk

Abstract

The macromolecule glycogen is the major glucose reserve in eukaryotes and defects of glycogen metabolism and structure lead to glycogen storage diseases and neurodegeneration. Glycogenesis begins with self-glucosylation of glycogenin (GN), which recruits glycogen synthase (GS). GS is activated by glucose-6-phosphate (G6P) and inactivated by phosphorylation, but how these opposing processes are coupled is unclear. We provide the first structure of phosphorylated human GS-GN complex revealing an autoinhibited GS tetramer flanked by two GN dimers. Phosphorylated N- and C-terminal tails from two GS protomers converge to form dynamic "spike" regions, which are buttressed against GS regulatory helices. This keeps GS in a constrained "tense" conformation that is inactive and more resistant to G6P activation. Mutagenesis that weaken the interaction between the regulatory helix and phosphorylated tails leads to a moderate increase in basal/unstimulated GS activity, supporting the idea that phosphorylation contributes to GS inactivation by constraining GS inter-subunit movement. We propose that multivalent phosphorylation supports GS autoinhibition through interactions from a dynamic "spike" region, thus allowing a "tuneable rheostat" for regulating GS activity. Our structures of human GS-GN provide new insights into the regulation of glycogen synthesis, facilitating future studies of glycogen storage diseases. 


\section{Introduction}

39 Glycogen is a branched polymer of glucose that functions as a primary energy store in eukaryotes. In its mature form, the glycogen particle can comprise up to $~ 50,000$ glucose units that can be rapidly utilized when glucose levels are low. Glycogen is stored predominantly in the muscle and liver cells, and to a lesser extent in other organs and tissues including kidney, brain, fat and heart ${ }^{1}$.

Glycogen is synthesized through the cooperative action of three enzymes: glycogenin (GN), glycogen synthase (GS) and glycogen branching enzyme (GBE) ${ }^{2}$. GN initiates the process via auto-glucosylation of a conserved tyrosine residue, producing a primer glucose chain of 8-12 resides connected by $\alpha-1,4$-linkages ${ }^{3}$. This glycogen initiating particle is further extended by GS after its recruitment by the $\mathrm{GN}$ C-terminus $\left(\mathrm{GN}^{34}\right)$ allowing the addition of glucose residues using $\alpha-1,4$-linkages ${ }^{4,5}$. GBE introduces $\alpha-1,6$-linkages every $6-8$ residues to the growing glycogen molecule, thus creating the final globular structure containing $\mathrm{GN}$ at the centre $\mathrm{e}^{2,6}$. Glycogen exists as a population of molecules with varying sizes $(10-290 \mathrm{~nm})$ in different tissues and species, although the importance of this variability is not well understood ${ }^{1,7}$.

Glycogen synthesis and breakdown are tightly regulated processes, and thus dysregulation of enzymes involved in glycogen metabolism contributes to glycogen storage diseases Excessive and/or abnormal glycogen is a common characteristic in most GSDs. Pompe disease (GSDII) is caused by deficiency of acid- $\alpha$-glucosidase, resulting in accumulation of lysosomal glycogen and consequent lysosomal destruction and dysfunction ${ }^{9}$. Lafora disease is a fatal neurodegenerative condition, of which the hallmark is Lafora bodies, which are hyperphosphorylated and poorly branched, insoluble glycogen deposits ${ }^{10}$. Furthermore, loss of GS-GN interaction results in muscle weakness and cardiomyopathy ${ }^{11}$.

Studies using mouse models have found inhibition of glycogen synthesis, and in particular by reducing GS activity, to be beneficial for multiple GSDs ${ }^{12,13}$. To date there is no structure of the GS-GN complex and no structure of human GS. Since inhibition of GS activity is potentially beneficial for GSD patients, obtaining a human GS-GN structure and understanding how GS is regulated is instrumental in developing new therapeutics.

$71 \mathrm{GN}$ is found in two isoforms, GN1 and GN2, encoded by the GYG1 and GYG2 genes 72 respectively. While GYG1 is widely expressed, GYG2 is restricted to the liver, pancreas and 
heart ${ }^{14,15}$. GN belongs to the GT8 family of glycosyltransferases, containing a glycosyl transferase A (GT-A) fold with a single Rossmann fold domain at the N-terminus, which is essential for binding of the glucose donor uridine diphosphate glucose (UDP-G) ${ }^{16-18}$. The Cterminus comprises a highly conserved region of $\sim 34$ residues $\left(\mathrm{GN}^{34}\right)$ which is the minimal targeting region for binding $\mathrm{GS}^{5,19}$. Other interaction interfaces have been suggested ${ }^{20}$, however, further investigation into the full-length complex is required to determine any additional interaction interfaces. The area between the $\mathrm{N}$-terminal catalytic domain and $\mathrm{C}$ terminal GS binding motif is a linker region that is variable in sequence and in length (Fig. 1a and Supplementary Fig. 1).

82

GS is also found as two isoforms, GS1 and GS2, encoded by the GYS1 and GYS2 genes respectively. These are differentially expressed, with GYS1 being expressed predominantly in skeletal muscle and most other cell types where glycogen is present, while GYS2 is expressed exclusively in the liver ${ }^{21-23}$. Eukaryotic GS belongs to the GT3 family of glycosyltransferases with a GT-B architecture comprising an N-terminal and a C-terminal Rossmann fold domain, with an interdomain cleft that contains the active site ${ }^{16,24}$. GS is the rate limiting enzyme in glycogen biosynthesis and as such its activity is tightly regulated ${ }^{25}$. GS is inactivated by covalent phosphorylation at numerous $\mathrm{N}$ - and C-terminal sites (Fig. 1a), and is allosterically activated by glucose-6-phosphate (G6P) binding and/or dephosphorylation ${ }^{2,26,27}$. Human GS (HsGS) phosphorylation sites lie at the $\mathrm{N}$-terminus (sites 2 and 2a) and C-terminus (sites 3a, $3 b, 3 c, 4,5,1 a, 1 b)$, and phosphorylation occurs in a hierarchical fashion, whereby the phosphorylation of a specific site is the recognition motif for subsequent phosphorylation ${ }^{28,29}$ (Fig. 1a and Supplementary Fig. 2). How the metazoan GS is inhibited is not clear and while coupling of G6P binding and phosphorylation has been described for the yeast GS paralogues ${ }^{30}$ no structural information of the phosphorylated version of the enzyme exists.

The complex interplay between allosteric activation and inhibitory phosphorylation is not yet fully understood, at least in part because of the lack of structural data for the full GS-GN complex. Although a binary GS-GN complex was co-purified over 30 years ago ${ }^{3}$, we have yet to confirm the stoichiometry of this complex and identify precisely how the two proteins cooperate to make glycogen.

Here, we report the structural and functional analysis of the full-length human GS-GN complex and the cryo-EM structure of phosphorylated HsGS. The structure reveals phosphoregulatory elements form a flexible inter-subunit "spike" region emanating from two GS protomers which help to keep GS in an inactive conformation through interactions of phosphorylated S641 (site 
110 3a) with arginine residues from GS regulatory helices (arginine cradle). Moreover, low 111 resolution maps of GN bound to GS reveal two flexible GN dimers coordinating a GS tetramer, 112 providing new insights into the stoichiometry and the conformational plasticity of this enzyme 113 complex. Collectively, these results shed light on the regulation of glycogen biosynthesis and 114 the inner workings of how GS and GN cooperate to synthesize glycogen.

\section{Results}

\section{The GS-GN complex forms an equimolar 4:4 complex}

120 To characterize the synthesis of glycogen by the GS-GN complex, we expressed and purified 121 human full length GS1 and GN1 in insect cells. Consistent with previous reports, co122 expression of GS with GN resulted in improved production yields over the expression of GS 123 alone ${ }^{31,32}$. Purification of the wild-type (WT) complex resulted in a highly glucosylated sample, 124 as shown by a smear on an SDS-PAGE gel corresponding to glucosylated GN detected by 125 Coomassie and periodic acid-Schiff (PAS) staining (Fig. 1c). Mutation of the GN auto126 glucosylating tyrosine $195^{33,34}$ to a phenylalanine (Y195F), resulted in a non-glucosylated GN 127 species, as shown by a single band for GN migrating at the expected size ( $37.5 \mathrm{kDa})$ on an 128 SDS-PAGE gel and absence of glucosylated species after PAS staining (Fig. 1b and 1c).

130 To determine the stoichiometry of the GS-GN complex, we first performed mass photometry 131 analysis of GS-GN and GS-GN(Y195F) mutant complexes, which enables mass 132 measurements of single molecules in solution. Mass photometry measurements of the GS$133 \mathrm{GN}(\mathrm{Y} 195 \mathrm{~F})$ complex showed a predominant molecular weight of $473+/-43 \mathrm{kDa}$, which is 134 suggestive of a 4:4 stoichiometry (calculated mass of $485 \mathrm{kDa}$ ) (Fig. 1d). Analysis of the GS$135 \mathrm{GN}(\mathrm{WT})$ sample identified a species with an average molecular weight of 534 +/- $110 \mathrm{kDa}$ and 136 the measured peak was broader than the non-glucosylated species (Fig. 1d). The increase in 137 molecular weight and distribution compared to the Y195F complex can be attributed to the 138 glucosylation associated with the WT complex. 
a

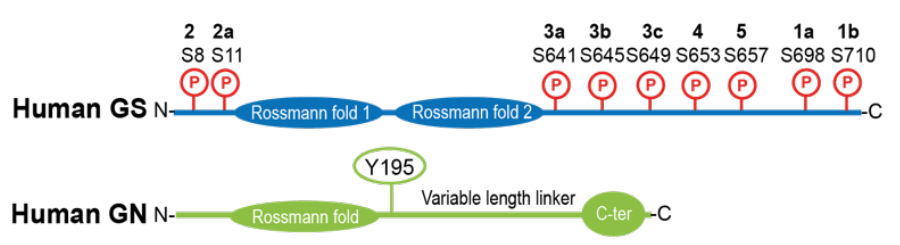

C
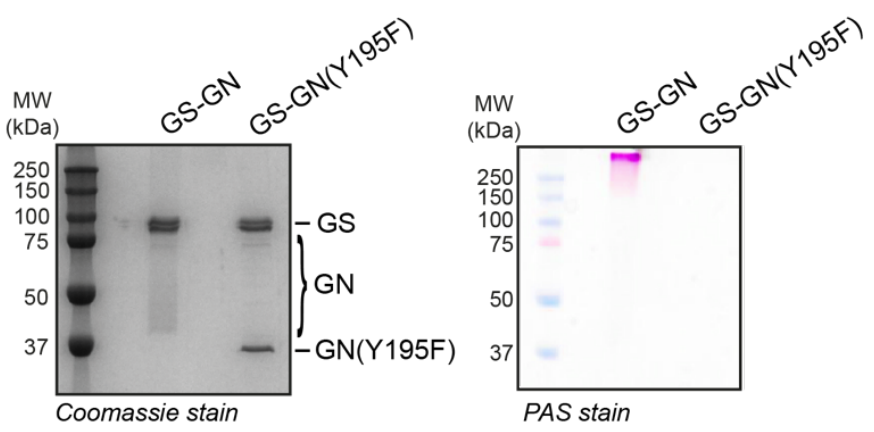

e

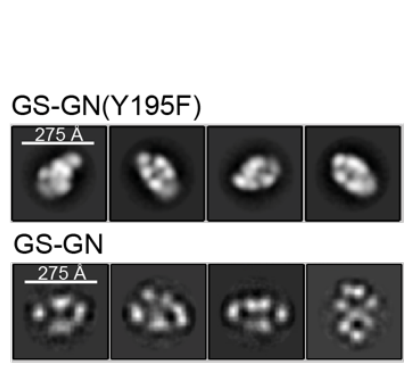

$\mathbf{f}$

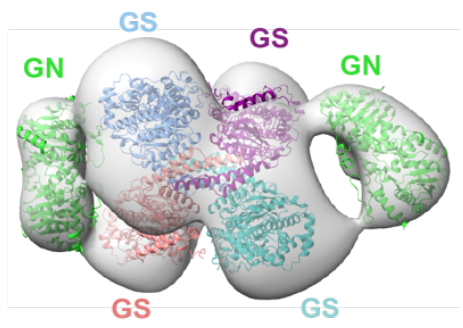

b
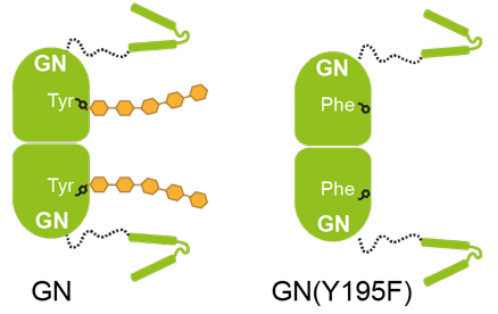

d
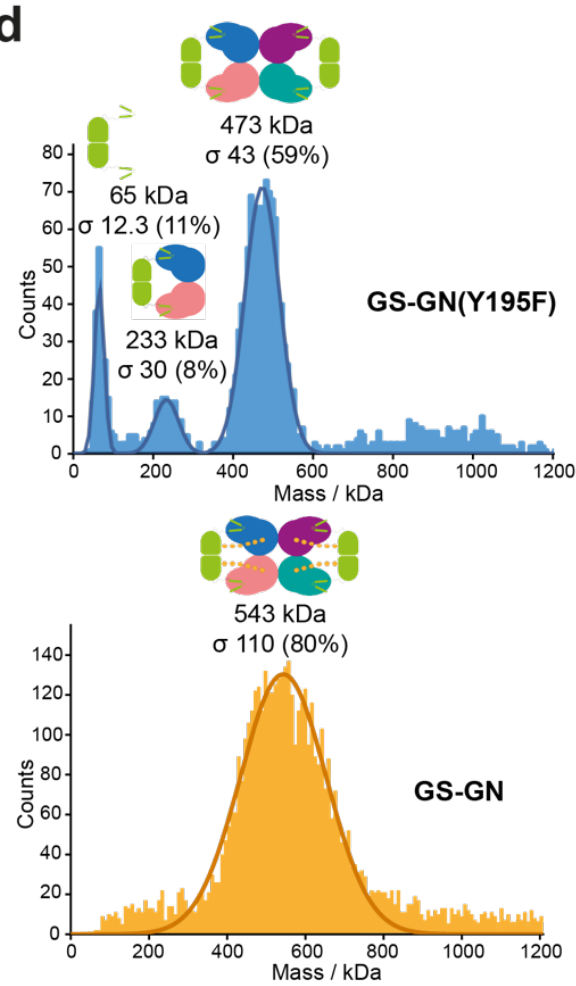

Fig. 1 Structural analysis of the full-length GS-GN complex

a Domain architecture of human GS (top) and human GN (bottom). Known in vivo phosphorylation sites of GS are shown in red and are labelled with residue number and classical nomenclature (in bold). GN tyrosine 195 that becomes auto-glucosylated and mutated to a phenylalanine (Y195F) in this study is indicated. Not to scale. b Cartoon representation of GN WT and Y195F. c SDS-PAGE analysis of GS-GN WT and Y195F complexes (left) and periodic acid-Schiff (PAS) staining of both complexes (right). d Mass photometry of GS-GN(Y195F) (top) and WT complex (bottom). Expected stoichiometry for each peak is indicated. e Selected 2D class averages after negative-stain electron microscopy (nsEM) analysis of GS-GN(Y195F) (top) and WT (bottom), revealing a GS tetramer and two GN dimers. f nsEM final map (C1 symmetry, 22 $\AA$ ) is shown in transparent surface, with fitted human GN crystal structure (PDB ID 3T7O) and human GS cryo-EM structure (reported here).

To understand how GS and GN interact within the GS-GN complex, and to reveal the overall shape of the complex we performed negative stain electron microscopy (nsEM) of both the WT and Y195F complexes. 2D class averages show two GN dimers, one on either side of a GS tetramer, for both complexes (Fig. 1e). Final 3D maps for both complexes are consistent with the 2D classes, and the density can accommodate a GS tetramer and two GN dimers (Fig. 1f). This nsEM confirms a 4:4 stoichiometry and is consistent with previous findings showing that glucose chains are not essential for the GS-GN interaction and that GS can 
interact with four GN C-terminal peptides simultaneously ${ }^{5,19}$. Surprisingly, GN dimers do not engage GS dimers in an identical fashion, with one GN tilted slightly towards one of the GS protomers (Fig. 1f). Collectively, these results confirm that the GS-GN complex has a 4:4 stoichiometry where two GN dimers can engage a single GS tetramer. This provides the first glimpse of the GS-GN complex structure and explains how GS can extend glucose chains from two GN dimers simultaneously.

\section{The structure of human GS reveals a dynamic "spike" region}

Previous attempts to crystallise full length GS in complex with full length GN were unsuccessful $1^{19}$ and led us to pursue structural analysis using cryo-electron microscopy (cryoEM). NsEM indicated that the position of each GN dimer is different suggesting flexibility of GN in the complex. Cryo-EM analysis of the GS-GN(Y195F) complex confirmed this GN flexibility, evident from the lack of GN signal in 2D class averages (Supplementary Fig. 4a) and subsequent 3D maps. Although we could detect GN presence after data processing without the application of symmetry averaging (Supplementary Fig. 5a and 5b), it was not possible to trace the connecting residues between the GN globular domain and the C-terminal

$173 \mathrm{GN}^{34}$ region that binds GS. To gain a higher resolution structure for the human GS, we applied 174 D2 symmetry and achieved a global resolution of $4 \AA$ (Fig. 2a, Supplementary Fig. 4, 175 Supplementary Table 1). The reconstruction revealed a tetrameric arrangement of HsGS in agreement with the crystal structure of C. elegans (CeGS) and yeast (yGS) (Supplementary Fig. 6a). Density for the C-terminal GS interacting region of GN is sufficient for a model of the human GN C-terminus to be placed and this is consistent with the CeGS-GN ${ }^{34}$ crystal structure (Fig. 2a and Supplementary Fig. 6b). Structural analysis of the HsGS-GN(WT) complex revealed a $6 \AA$ map of GS and comparing this to the GS structure from HsGS-GN(Y195F) complex reveals no differences at this resolution (Supplementary Fig. $\mathbf{5 c}$ and $\mathbf{5 d}$ ).

Notably, the EM density maps show density for an inter-subunit apparatus containing the GS phosphoregulatory regions. This occupies the space where the $\mathrm{N}$ - and $\mathrm{C}$ - terminal regions of two adjacent GS protomers meet and traverse away from the core (Fig. 2b). Local resolution analysis shows that this area is resolved at lower resolution than the core of the GS tetramer

187 (Supplementary Fig. 4b). Analysis of this $\sim 25 \mathrm{kDa}$ region by focussed 3D classification (without applying symmetry) reveals that the region is highly flexible, as seen by the various different conformations, explaining the lower resolution and uninterpretable density (Fig. 2c and Supplementary Fig. 4f). Interestingly, these "spike" regions were present in all the refined classes, and suggests that GS exists as a continuum of structures with a core inactive 
bioRxiv preprint doi: https://doi.org/10.1101/2021.11.14.468517; this version posted November 14, 2021. The copyright holder for this preprint (which was not certified by peer review) is the author/funder, who has granted bioRxiv a license to display the preprint in perpetuity. It is made available under aCC-BY-NC-ND 4.0 International license.

tetramer and "dynamic spikes" buttressed on either side, thus constraining the opening of GS to an active tetramer.

195 To explore the flexibility and mobility of GS, we performed 3D variability analysis ${ }^{35}$ using cryoSPARC $^{36}$. The dynamic movements of the "spike" region and concurrent movements of the GS tetramer are highlighted in Movie S1. While some flexibility was observed within each GS protomer, an opening of the GS tetramer towards an active, relaxed state was not observed, highlighting the crucial role of the spike regions in maintaining an inactive, tense

a

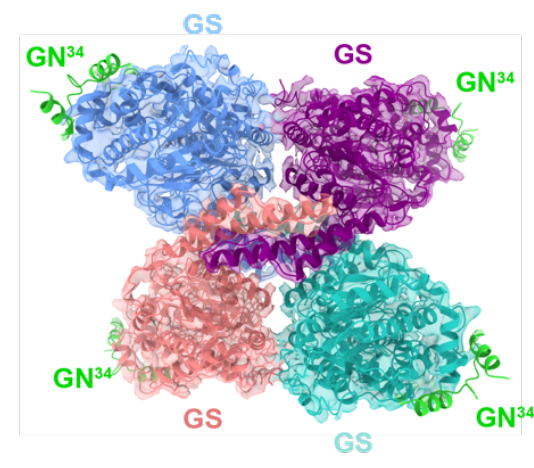

C
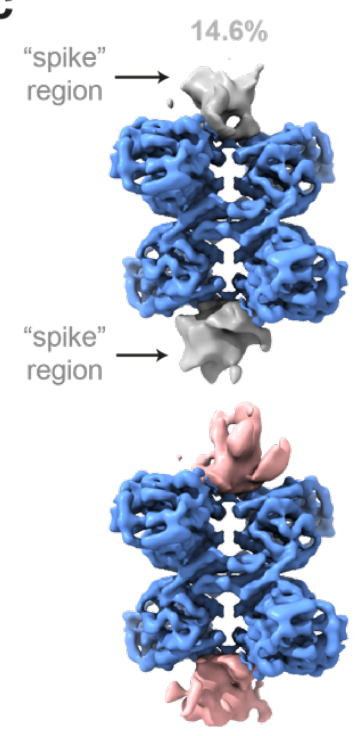

$10.4 \%$
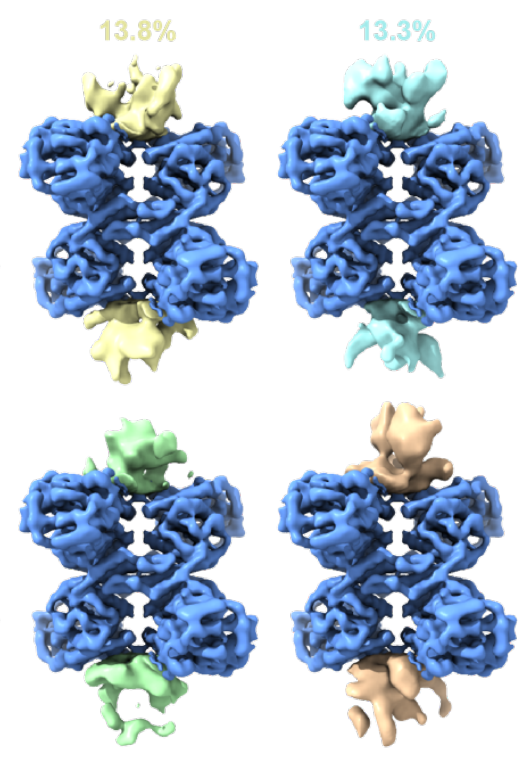

$7.8 \%$

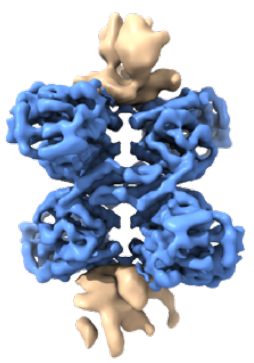

$7.4 \%$ b
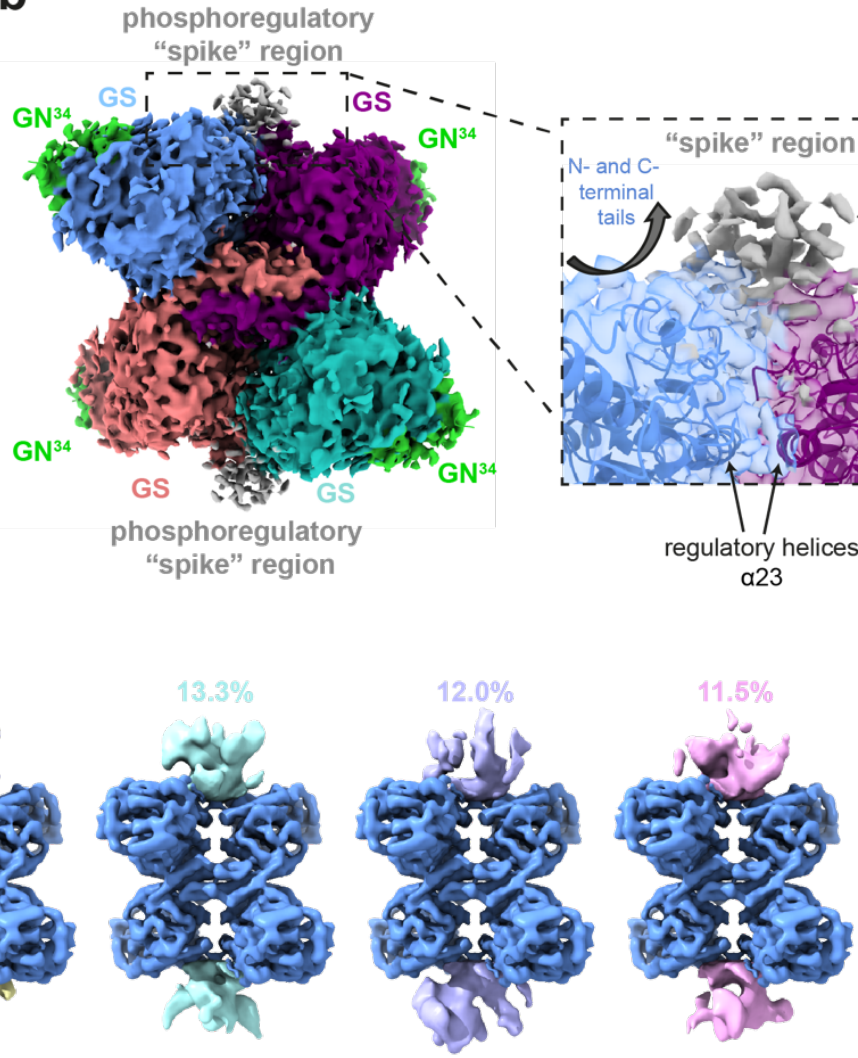

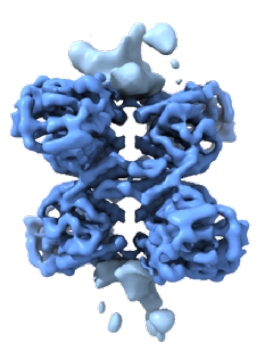

$5.4 \%$

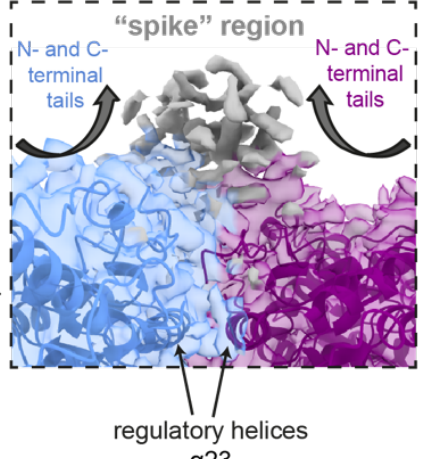

a23

Fig. 2 Cryo-EM structure of human GS-GN complex

a Human GS cartoon model shown in ribbons and a $4 \AA$ reconstructed cryo-EM map shown in transparent surface and coloured by corresponding chain. A model of the last 34 residues of the $C$ terminus of human $\mathrm{GN}\left(\mathrm{GN}^{34}\right)$ is displayed in green. b Cryo-EM density map shown at a lower threshold (left). The $\mathrm{N}$ - and $\mathrm{C}$-terminal tails of two protomers converge and form the "spike" region (right). c Focussed 3D classification of the phosphoregulatory "spike" region reveals flexibility of this inter subunit domain. 3D classes and percentage population are indicated with different colours and the 3D refined map of the core region is coloured blue. 


\section{Phosphorylated human GS is in the inactive state}

203 GS is regulated by both allosteric activation by G6P and inhibition via phosphorylation of its $204 \mathrm{~N}$ - and C-terminal tails ${ }^{2}$. Mechanistic and structural studies of the yGS have elegantly 205 dissected its allosteric activation by G6P, whereby G6P binding induces large rotational and 206 translational movements, leading to a more accessible active site for the growing glycogen 207 chain to be fed into GS for elongation ${ }^{27,30}$. However, the mechanisms governing GS activation 208 by G6P and inactivation by phosphorylation, and how these opposing regulatory pressures 209 are coupled at the molecular level remains unknown.

211 An advantage of expressing HsGS in insect cells is that these preparations are heavily 212 phosphorylated. We mapped phosphorylation sites by tandem mass spectrometry (MS/MS) 213 following proteolysis with either trypsin, chymotrypsin or elastase resulting in a sequence 214 coverage of $97 \%$, which is higher than the $73 \%{ }^{31}$ and $65 \%{ }^{32}$ sequence coverage achieved in 215 previous studies (Table 1 and Supplementary Fig. 7b). This analysis identified canonical sites 2, 3a, 3b, 4 and 5 (S8, S641, S645, S653, S657), and also non-conventional sites (S412, S652, S727, S731). In addition, we could detect HsGS site 2 (S8) phosphorylation for the first time in a recombinant enzyme preparation. Together, these results show that expression in insect cells is sufficient to achieve a high level of phosphorylation providing suitable enzyme preparations for understanding inactive GS.

As expected, our highly phosphorylated enzyme has a low basal (-G6P) level of activity (Fig. 3a). Protein phosphatase 1 (PP1) and lambda phosphatase (lambda PP) treatment resulted in faster migration of GS in an SDS-PAGE and also a reduction in signal for specific phosphorylation site antibodies (Fig. 3b and Supplementary Fig. 7a). We observed over 10fold increase in basal activity when GS is dephosphorylated by both PP1 and lambda PP (Fig. 3a and 3b). Both the phosphorylated and dephosphorylated GS forms were similarly active after addition of G6P (Fig. 3a), which is consistent with studies using GS from endogenous sources $^{37,38}$. 
Table 1: Summary of GS phosphorylation site analysis. LTP and HTP refer to low throughput site determination (methods other than mass spectrometry) and high throughput analysis (mass spectrometry only), respectively ${ }^{39}$

\begin{tabular}{|c|c|c|c|c|c|c|}
\hline Site & Sequence & Peptide modifications & ptmRS Score & MASCOT score & Enzyme & PhosphoSite \\
\hline $\mathrm{s} 8$ & PLNRTLSMS & S7(Phospho) & S7: 100 & 40 & Elastase & 15 LTP \& 1 HTP \\
\hline S8 & PLNRTLsMS & S7(Phospho); M8(Oxidation) & S7: 100 & 31 & Elastase & \\
\hline s8 & sMSSLPGLEDW & S1(Phospho) & S1: 99.71 & 13 & Chymotrypsin & \\
\hline S412 & ESLLVGSLPDMNKMLDKEDF & S7(Phospho) & S7: 100 & 31 & Chymotrypsin & 17 HTP \\
\hline S412 & ESLLVGsLPDMNKML & M14(Oxidation) & S7: 100 & 23 & Chymotrypsin & \\
\hline $\mathrm{S} 641$ & QGYRYPRPAsVPPSPS & S10(Phospho) & S10: 99.99 & 20 & Elastase & 32 LTP \& 30 HTP \\
\hline $\mathrm{S} 641 \& \mathrm{~S} 645$ & QGYRYPRPAsVPPSPS & S10(Phospho); S14(Phospho) & S10: $100 ;$ S $14: 100$ & 24 & Elastase & (645) 21 LTP \& 39 HTP \\
\hline S652 & RHsSPHQSEDEEDPRNGPL & S3(Phospho) & S3: 99.78 & 34 & Elastase & 15 HTP \\
\hline S657 & RHssPHQsEDEEDPRNGPL & S3(Phospho); S4(Phospho); S8(Phospho) & S8: 100 & 19 & Elastase & (653) 13 LTP \& 27 HTP \\
\hline $\mathrm{S} 652 \& \mathrm{~S} 657$ & RHsSPHQsEDEEDPRNGPL & S3(Phospho); S8(Phospho) & S3: 99.67; S8: 100 & 53 & Elastase & (657) 14 LTP \& 50 HTF \\
\hline S727 & RNSVDTATSSSLSTPSEPLSPTSSLGEER & S20(Phospho) & S20: 100 & 66 & Trypsin & 1 LTP \& 22 HTP \\
\hline S727 & STPSEPLSPTSSL & S8(Phospho) & S8: 99.63 & 20 & Chymotrypsin & \\
\hline $\mathrm{S} 731$ & STPSEPLSPTSLLGEERN & S12(Phospho) & S12: 99.79 & 73 & Chymotrypsin & 6 HTP \\
\hline S731 & TPSEPLSPTSsL & S11(Phospho) & S11: 100 & 22 & Elastase & \\
\hline $\mathrm{S} 727 \& \mathrm{~S} 731$ & NSVDTATSSSLSTPSEPLSPTSSLGEER & S19(Phospho); S23(Phospho) & 99.52 & 60 & Trypsin & see above \\
\hline S727 \& S731 & $\begin{array}{l}\text { STPSEPLSPTSSLGEERN } \\
\text { STS }\end{array}$ & S8(Phospho); S12(Phospho) & S8: $100 ;$ S12: 100 & 42 & Chymotrypsin & see above \\
\hline
\end{tabular}

\section{GS contains a dynamic and tuneable phosphoregulatory apparatus}

242 Comparisons between human, C. elegans and yeast GS structures are consistent with the 243 human structure in the inactive state. Each HsGS protomer shows a closed conformation of 244 its active site, and a regulatory loop, that only becomes ordered upon G6P binding, is 245 disordered in the human structure (Supplementary Fig. 8). Previous studies have suggested 246 that phosphorylated tails may be able to engage the G6P binding site and directly compete 247 with G6P. However, our EM density maps show no extra density within the G6P binding site 248 (Supplementary Fig. 8a). Thus, we see no evidence to support the hypothesis that the 249 phosphorylated tails directly interact with residues lining the G6P pocket to directly compete 250 with G6P binding. Instead, we posit that the phosphoregulatory regions indirectly affect G6P binding by constraining the opening and closing of the GS tetramer.

253 When comparing HsGS to previous yeast crystal structures, the distance between regulatory 254 helices ( $\alpha 23$ in humans, $\alpha 22$ in yeast) in adjacent monomers changes according to the activity 255 state of GS (Fig. 3c). Using the C $\alpha$ atoms on residue 591 on chain A and residue 580 on chain $B$ (and the corresponding in the yeast proteins) the helices are furthest apart, at $16 \AA$, when G6P is bound and GS is in its high activity state, as there is better access for accepting the substrate ${ }^{27,30}$. When no G6P is bound and there is no phosphorylation, GS is in the basal state and the helices lie $11 \AA$ apart. In a yGS structure of a mimic of the inhibited state, where residues $\mathrm{R} 589$ and $\mathrm{R} 592$ were mutated to Ala, the helices are closest together, at $8 \AA$, leading to the closure of the $\mathrm{N}$-terminal domain and thus acceptor access is hindered ${ }^{30}$. HsGS produced in insect cells is phosphorylated ${ }^{31,32}$ (Fig. 3b, Supplementary Fig. 7) and the

263 helices lie $8 \AA$ apart, thus confirming that we have reconstructed the first phosphorylated, auto264 inhibited structure (Fig. 3c).

265 The $\mathrm{N}$ - and C-terminal tails of each GS protomer lie parallel to each other, and travel side by 266 side along the GS tetrameric core to reach centre. Here, they meet the $\mathrm{N}$ - and C-terminal tails 
267 from an adjacent GS protomer, which have travelled from the opposite direction (Fig. 3d).

268 From the electron density map generated without the application of symmetry averaging, it

269 appears that one C-terminal tail disengages with the GS core earlier than the other C-terminal

270 tail from the adjacent chain. The $\mathrm{C}$-terminal tail from chain $\mathrm{B}$ continues to travel further across

271 the regulatory helices than chain A, prior to traversing away from the core to form the "spike"

272 region (Fig. 3d). This allows chain $B$ to engage with the regulatory helices $\alpha 23$, specifically

273 interacting with residues R588 and R591, which come from two GS protomers to form a

274 positively charged pocket we have termed the "arginine cradle" (Fig. 3e). Judging by the

275 distance from the last discernible residue of the C-terminal tail and the available cryo-EM

276 density we have modelled a phosphorylated S641 (pS641) in chain B in interacting distance

277 with the arginine cradle (Fig. 3d). The position of S641 is consistent with the AlphaFold ${ }^{40}$

278 predicted model of HsGS (Supplementary Fig. 8d) suggesting that this interaction may also

279 be possible in the non-phosphorylated state, although the negative charge on the phosphate

280 group would naturally provide stronger interactions with the positively charged arginine cradle.

281 S641 is a major phosphorylation site involved in activity regulation, and interaction of pS641

282 with the arginine cradle from helix $\alpha 23$ provides a crucial activity switch mechanism from a

283 tense (phosphorylated) state to a relaxed (G6P-bound) state. The involvement of helices $\alpha 23$

284 which also interacts with G6P via the nearby arginine residues R582 and R586

285 (Supplementary Fig. 8a) is significant, since this could provide a possible explanation

286 between G6P-binding and its ability to override inactivation by phosphorylation. 
bioRxiv preprint doi: https://doi.org/10.1101/2021.11.14.468517; this version posted November 14,2021 . The copyright holder for this

preprint (which was not certified by peer review) is the author/funder, who has granted bioRxiv a license to display the preprint in perpetuity. It is made available under aCC-BY-NC-ND 4.0 International license.

a

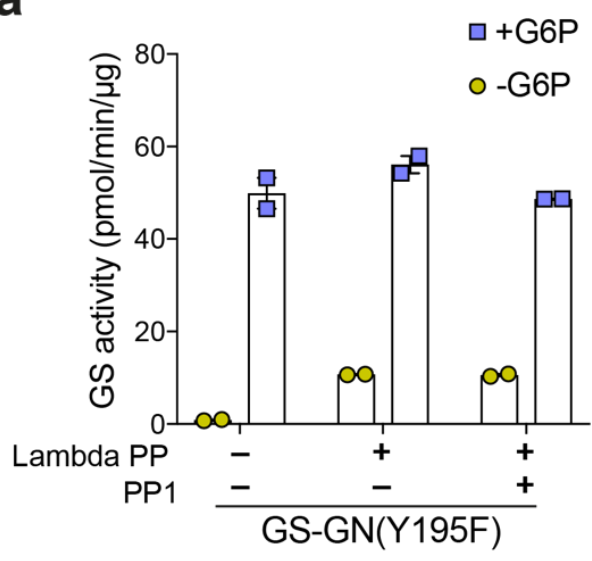

b

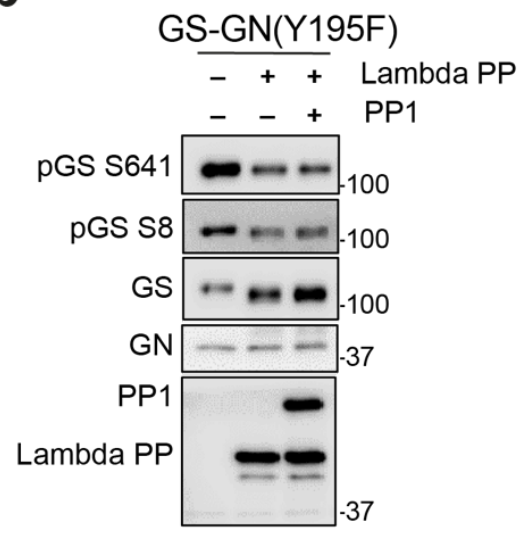

C

HsGS

Inhibited state

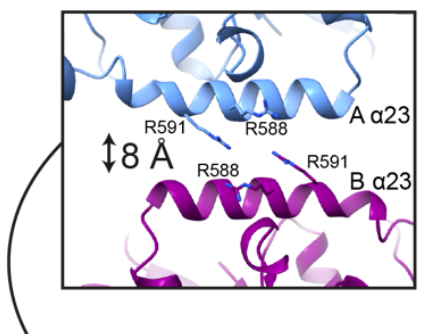

GS

chain A e

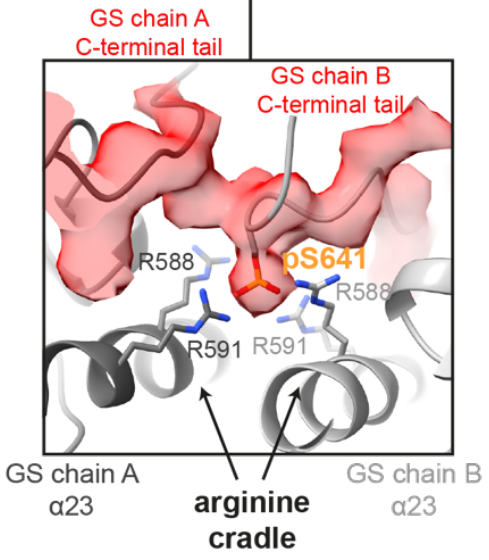

yGS

Mimic of inhibited state

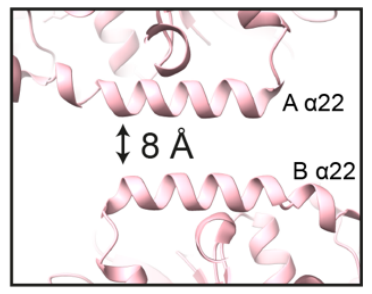

d

yGS

Basal state

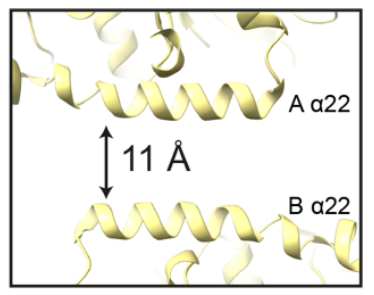

yGS

Activated state

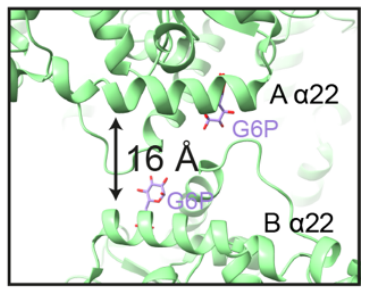

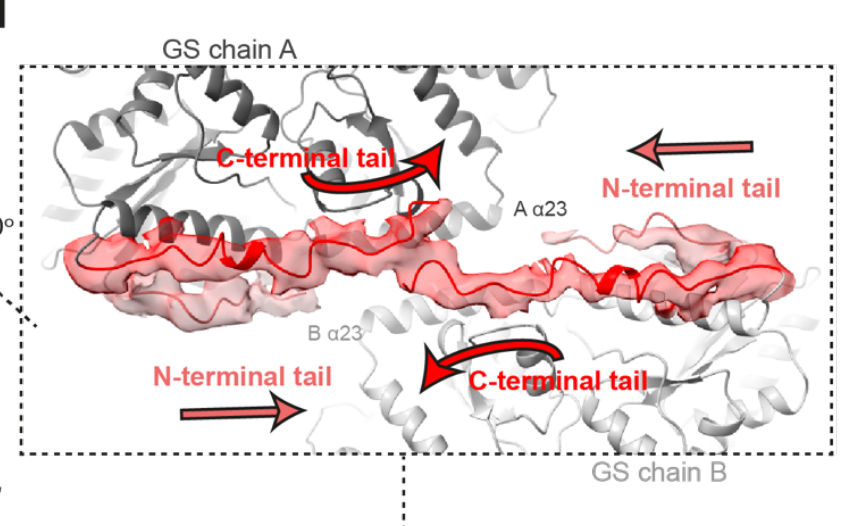

f GS chain $A$

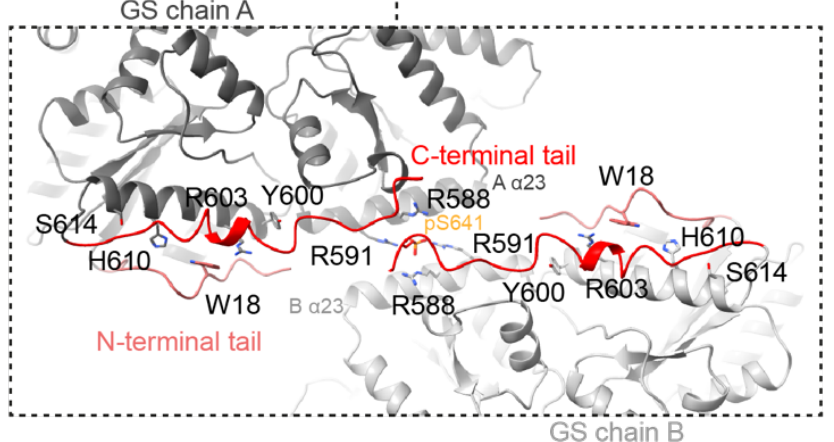




\section{Fig. 3 Dynamic and tuneable phosphoregulatory region of human GS}

a Activity of GS-GN(Y195F) with and without the addition of lambda phosphatase (lambda PP) and protein phosphatase 1 (PP1). Upon G6P saturation, GS reaches similar activity levels regardless of phosphorylation state. Data are mean + -- S.E.M. from $n=2$ and representative of two independent experiments. b Western blot for the indicated human GS phosphorylation sites and total GS. c Comparison of distances between regulatory helices of adjacent monomers of human GS (reported here), low activity inhibited mimic (PDB ID 5SUL), basal state (PDB ID 3NAZ) and G6P activated (PDB ID 5SUK) yeast crystal structure. Distances were measured from the $\mathrm{C} \alpha$ of residues 591 chain $\mathrm{A}$ and 580 chain $\mathrm{B}$ (and corresponding yeast residues). $\mathrm{d}$. The $\mathrm{N}$ - and $\mathrm{C}$ - terminal tails of one protomer lie parallel and move towards the bottom of the "spike" region, meeting the $\mathrm{N}$ - and $\mathrm{C}$ terminal tails of the adjacent GS protomer e Electron density for phosphorylated S641 (pS641) interacting with R588 and R591 on the regulatory helices $\alpha 23$. $f$ Residues that are interacting with the $\mathrm{N}$ - and $\mathrm{C}$-terminal tails that are mutated in this study are shown.

Aligning the structure of the C-terminal domain residues of human, yeast and C. elegans GS (residues 294-597 in HsGS, residues 283-598 in yGS and residues 312-679 in CeGS) revealed that helix $\alpha 3$ (human residues $85-96$ ) is shifted towards the C-terminal domain, compared to the yeast basal state. This N-terminal closure observed when comparing the yeast basal and mimic of the phosphorylated state crystal structures was suggested to contribute to the inhibited state of $\mathrm{GS}^{30}$. Consistent with these analyses, we observe an even bigger shift of helix $\alpha 3$ and a tight $\mathrm{N}$-terminal closure in the phosphorylated human GS (Supplementary Fig. 8c).

The position of the extreme $\mathrm{N}$-terminus is noticeably different in human and C. elegans GS structures compared to yeast (Supplementary Fig. $6 \mathbf{c}$ ). The majority of the first $\beta$ sheet in all structures is in the same place, however human residues before 26 (residue 7 in yeast) move in the opposing direction to yeast (Supplementary Fig. 6c). This positioning of the human GS $\mathrm{N}$-terminus is directed towards the regulatory helices ( $\alpha 23)$. Previous structural investigation of CeGS-GN ${ }^{34}$ suggested a hypothesis where phosphorylation could enable the $\mathrm{N}$-terminus to engage with regulatory helices ${ }^{19}$. Our human phosphorylated structure supports this hypothesis, although the current density does not allow model building before residue 14 . However, weak density for the $\mathrm{N}$-terminus is present showing that this region also interacts with the globular GS domain and regulatory helices, thus explaining $\mathrm{N}$-terminal phospho tail contribution to GS inactivation (Fig. 3d and Supplementary Fig. 6d). Collectively, our structural analyses support a model by which phosphorylated $\mathrm{N}$ - and C-terminal tails inhibit the GS tetramer by constraining a tense conformation through inter-subunit interactions.

312 Dislodging the GS phosphoregulatory regions

Due to the flexibility evident in the $\mathrm{N}$ - and C-terminal tails, we were unable to build 
316 beginning of the flexible phosphoregulatory "spike" region and residues from the GS "core 317 tetramer" which interact with this regulatory region (Fig. 3f). To investigate the relationship 318 between allosteric regulation and inhibitory phosphorylation and elucidate the mechanism of 319 inactivation, we mutated residues in GS that contact the beginning of the phosphoregulatory 320 region. We selected residues which are not involved in G6P binding and mutated these in 321 order to "dislodge" the regulatory tails (Fig. $\mathbf{3 f}$ and Supplementary Fig. 8e). If the phosphorylated tails are indeed holding GS in an inactivated state, weakening the interaction between the core tetramer and the "spike" regions should create an enzyme with higher basal activity in comparison to the WT. Consistent with our hypothesis, we observed a marginal increase in basal (-G6P) GS activity in R588A+R591A, Y600A, R603A, H610E and W18A mutants, that was reflective of the phosphorylated state at residues S8, S641 and S645 (Fig.

327 4a and $\mathbf{4 b}$ ). These mutants were unaffected in terms of GN co-purification and with the 328 exception of R588A+R591A mutant, they had similar melting $\left(T_{m}\right)$ profiles and oligomeric state 329 to the WT GS complexes (Fig. 4e, Supplementary Fig. 3b, 9a and 9b). All mutants except 330 Y600A could still be activated to similar levels to the WT upon addition of G6P (Fig. 4a).

332 Upon addition of PP1 and lambda PP, the GS mutants R588A+R591A, R603A and W18A 333 were more easily dephosphorylated at S641 and S8 than WT (Fig. 4d). For the W18A mutant, 334 this dephosphorylation by both lambda-PP and PP1 resulted in over a 20-fold increase in 335 basal activity, and also an approximately 3-fold increase in comparison to WT (Fig. 4c). The 336 GS R603A-GN(Y195F) mutant has a basal activity similar to WT upon dephosphorylation. 337 However, the robust dephosphorylation at S641 and S8 in GS R588A+R591A was not 338 associated with an increase in activity (Fig. 4c). As described above, R588 and R591 lie on 339 the regulatory helices and are also involved in inter-subunit interactions and form the arginine 340 cradle that interacts with pS641 (Fig. 3c). In the GS R588A+R591A double mutant we see 341 dissociation of the complex in mass photometry (Supplementary Fig. 9c). Therefore, the role 342 of these residues in stabilising the GS tetramer may be the cause for the lack of rescue of 343 activity upon dephosphorylation (Fig. 4c). Moreover, dephosphorylated GS had a markedly 344 lower $\mathrm{T}_{\mathrm{m}}\left(48^{\circ} \mathrm{C}\right)$ than WT or mutant GS supporting the idea that phosphorylation of the "spike" 345 regions strengthens the inter-subunit interactions within the tetramer and holding the enzyme 346 in the "tense" conformation. 
a

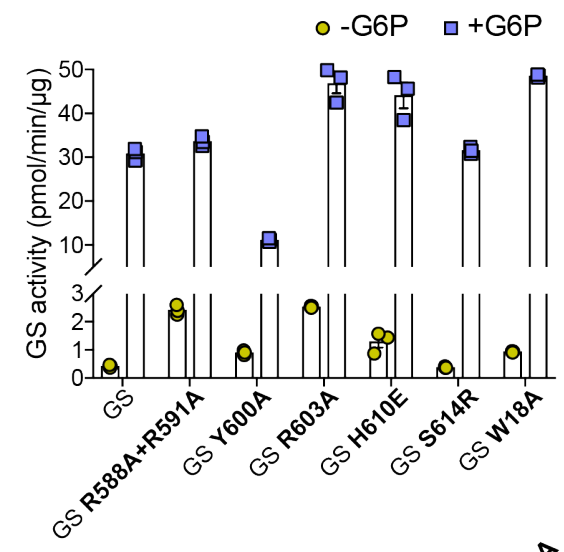

b
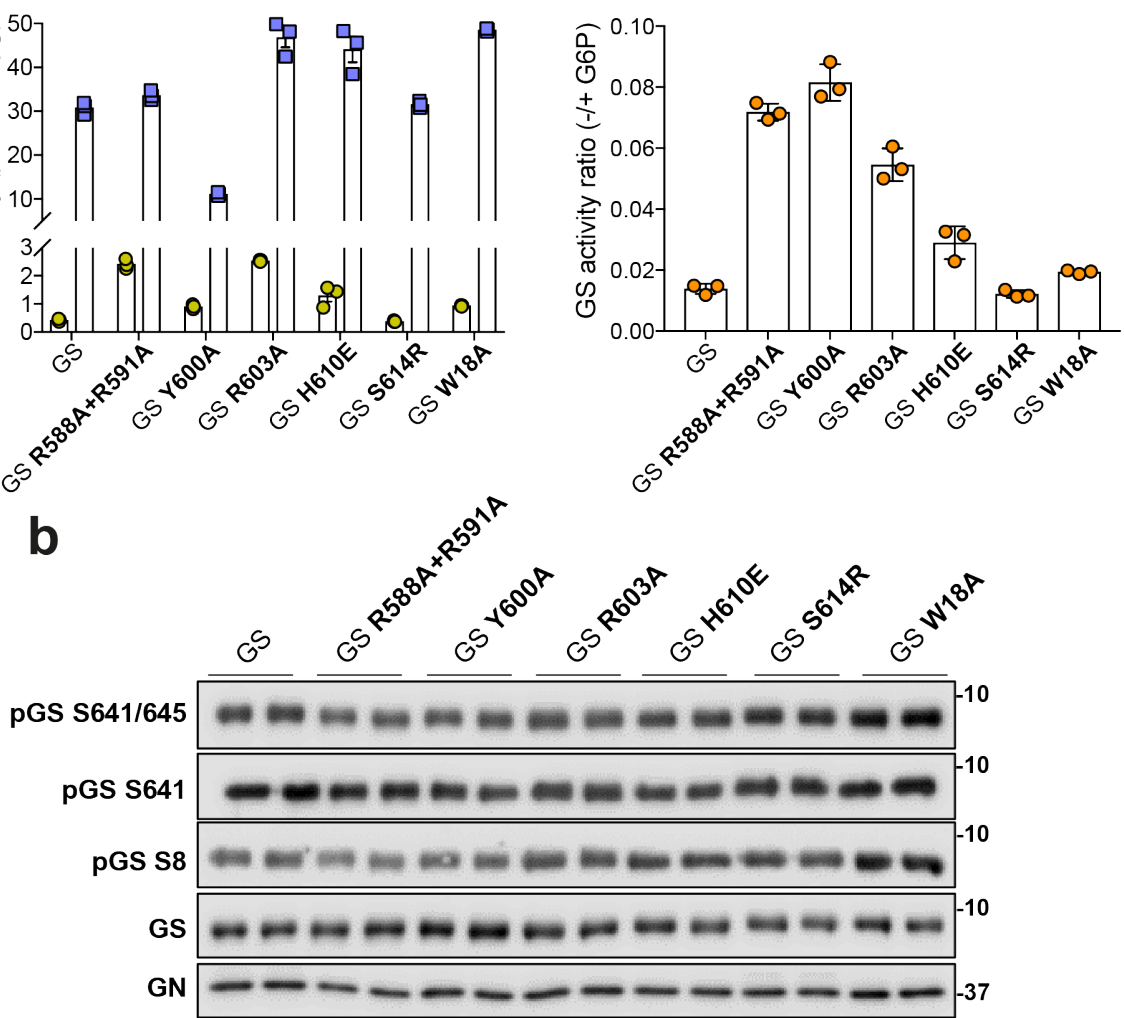

C

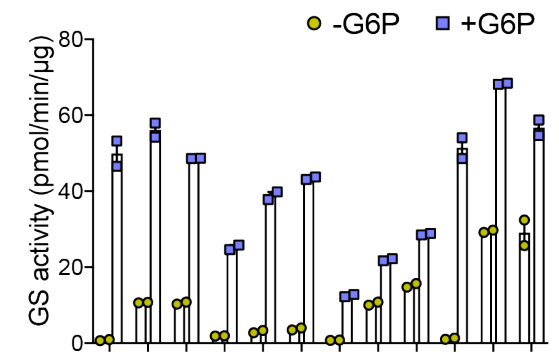

Lambda PP -+++++++++

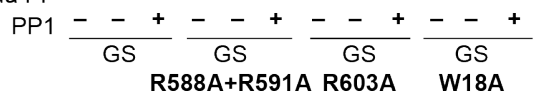

d

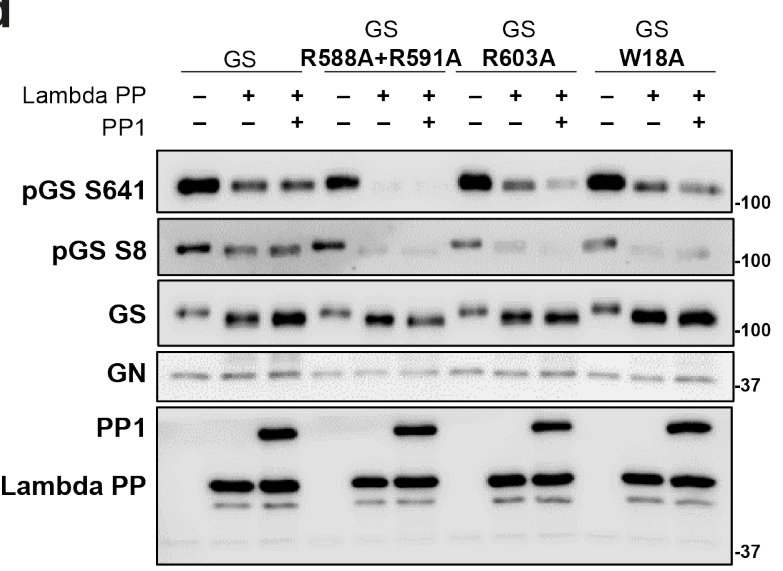

- No Phosphatase

口 + Lambda PP

$\nabla+$ Lambda PP + PP1

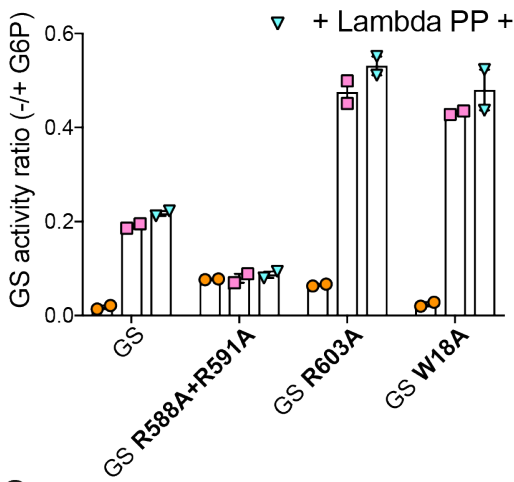

e

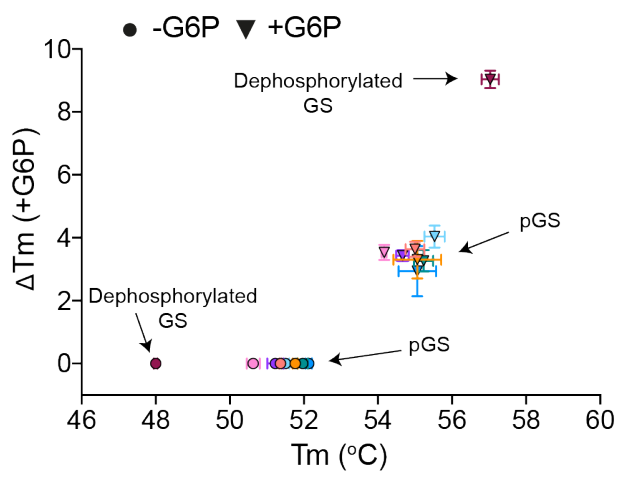

pGS pGSR588A+R591A pGSY600A pGSR603A pGSH610E

pGSW18A dephosphorylated GS 


\section{Discussion}

Fig. 4 Dislodging the GS phosphoregulatory region increases basal activity and increases accessibility for phosphatases

a Activity of GS WT and indicated mutants in the GS-GN(Y195F) complex in the presence and absence of G6P (left) and -/+ G6P activity ratio (right). Data are mean +/- S.E.M from $n=3$ and representative of two independent experiments. b Western blot for specific human GS phosphorylation sites S641/645, S641, S8, and total GS and GN. c Activity of phosphorylated and dephosphorylated GS WT and indicated mutants (left) and -/+ G6P activity ratio (right). Data are mean + - S.E.M from $n=2$ and representative of two independent experiments. $\mathbf{d}$ Western blots of GS WT and mutants in the GS-GN(Y195F) complex after dephosphorylation with PP1 and/or lambda phosphatase (lambda PP). e Melting temperature (Tm) of GS WT and mutants in the GS$\mathrm{GN}(\mathrm{Y} 195 \mathrm{~F})$ complex. Changes in melting temperature upon addition of $12.5 \mathrm{mM} \mathrm{G6P}\left(\Delta \mathrm{T}_{\mathrm{m}}=\right.$

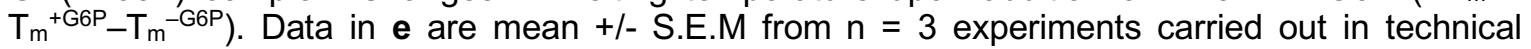
duplicates (dephosphorylated GS) and triplicates (WT and mutant GS).

Here, we provide structural and biochemical analysis of phosphorylated human GS in the fulllength GS-GN complex. Cryo-EM analysis reveals an inter-subunit flexible region which holds GS in an inactive conformation, shedding light on how phosphorylation of the $\mathrm{N}$ - and $\mathrm{C}$ terminal tails inhibit the enzyme (Fig. 5). In addition, negative stain EM maps reveal two GN dimers binding to a GS tetramer, explaining the conformational plasticity of this octameric enzyme complex and the inner workings of how GS-GN cooperate to synthesize glycogen (Fig. 1 and Fig. 5). The two GN dimers neighbouring a GS tetramer do not interact in an identical manner, with one GS dimer tilted closer towards GS in comparison to the other (Fig. 1f). This observed flexibility of GN shows the inherited dynamic nature within the complex which can be aided by the variable length linker connecting the catalytic domain and the Cterminal $\mathrm{GN}^{34}$ region that anchors GS. The precise functional relevance of this movement is yet to be explored, however the linker length was shown to govern glycogen particle size and molecular weight distribution in vitro ${ }^{19}$. Thus, the ability for $G N$ to orient and bind flexibly to GS may be a desirable feature facilitating the wide range of size and distribution of glycogen particles seen in multiple species and tissues ${ }^{4}$. Unfortunately, this inherent mobility also prevented a high-resolution reconstruction of GN structure and identification of any additional interfaces between GS and the globular catalytic domain on GN.

GS phosphorylation sites lie outside of the catalytic core on the $\mathrm{N}$ - and $\mathrm{C}$ - terminal tails (Fig. 1a and Supplementary Fig. 2). A mechanism by which phosphorylation could detach this $\mathrm{N}$ terminal region from its position, and move to interact with the arginine residues in the regulatory helices $\alpha 23$ was previously proposed ${ }^{19}$. Our structure of the phosphorylated state of GS supports this hypothesis, as the human $\mathrm{N}$-terminal tail lies in the same direction as $C$. elegans $\mathrm{N}$-terminal region situated against the regulatory helices, although the exact position differs (Supplementary Fig. 6c). More importantly, in human GS the C-terminus is 
responsible for the majority of the observable interactions with helix $\alpha 23$. R588 and R591 are not conserved in C. elegans (Supplementary Fig. 2), perhaps explaining the positional differences between human and $C$. elegans GS tails. This exchange of interactions between $\mathrm{N}$ - and $\mathrm{C}$-terminus suggests a functional redundancy between the multiple phosphorylation sites and a likely explanation for the dynamic nature of the "spike" regions.

The C-terminus of human GS runs parallel to the N-terminal tail, travelling alongside the GS core, prior to moving away from the core to form the "spike" region (Fig. 3d). Unexpectedly, the $\mathrm{C}$-terminal tail of adjacent protomers are not identical. One tail appears to disengage from the GS core sooner than the other. This leaves room for one tail to interact with the regulatory helix, specifically residue pS641 interacting with R588 and R591 on both protomers (Fig. 3d and 3e). S641 was identified as a site of phosphorylation by both phosphorylation mapping and western blotting (Table 1, Supplementary Fig. 7b and Fig. 3b). This negative moiety was coordinated by multiple arginine residues from the arginine cradle, and suggests a crucial role for phosphorylation in interacting with the regulatory helices. A negative charge from pS641 could be strengthening the interaction between two GS protomers, in comparison to basal and activated GS, thus constraining the GS tetramer and leading to an inactive enzyme (Fig. 3c).

The non-identical/different positions of the $\mathrm{N}$ - and $\mathrm{C}$-terminal tails as well as the flexibility of the "spike" region indicates a high level of coordination and communication both between the $\mathrm{N}$ - and C- termini of a single GS protomer, as well as between protomers (Fig. 2c and 3d). Having one tail buttressed against the regulatory helix and the other steering away from the core may allow interchanging of the tails based on the level of phosphorylation, revealing a significance for the existence of multiple phosphorylation sites. Having one tail more accessible to phosphatases may also aid/allow rapid dephosphorylation of GS, leading to an increase in GS activity and thus glycogen synthesis (Fig. 5).

Elucidating the role of the inter-subunit domain that house the phosphorylation sites through mutations that weaken the interactions between the core tetramer and the "spike" regions, resulted in basal activity equal to or higher than the WT, yet retaining activation by G6P (Fig.

408 4a). The GS Y600A mutant was not activated by G6P to the same extent as WT (Fig. 4b), 409 and although $Y 600$ does not directly bind to G6P, UDP or sugars ${ }^{27,41,42}$, it is possible that this hydrophobic residue is important for interdomain movements which are required for full GS activation. 


\section{Constrained "tense" GS}

Phosphorylated, auto-inhibited state
Open "relaxed" GS

Dephosphorylated, G6P activated state

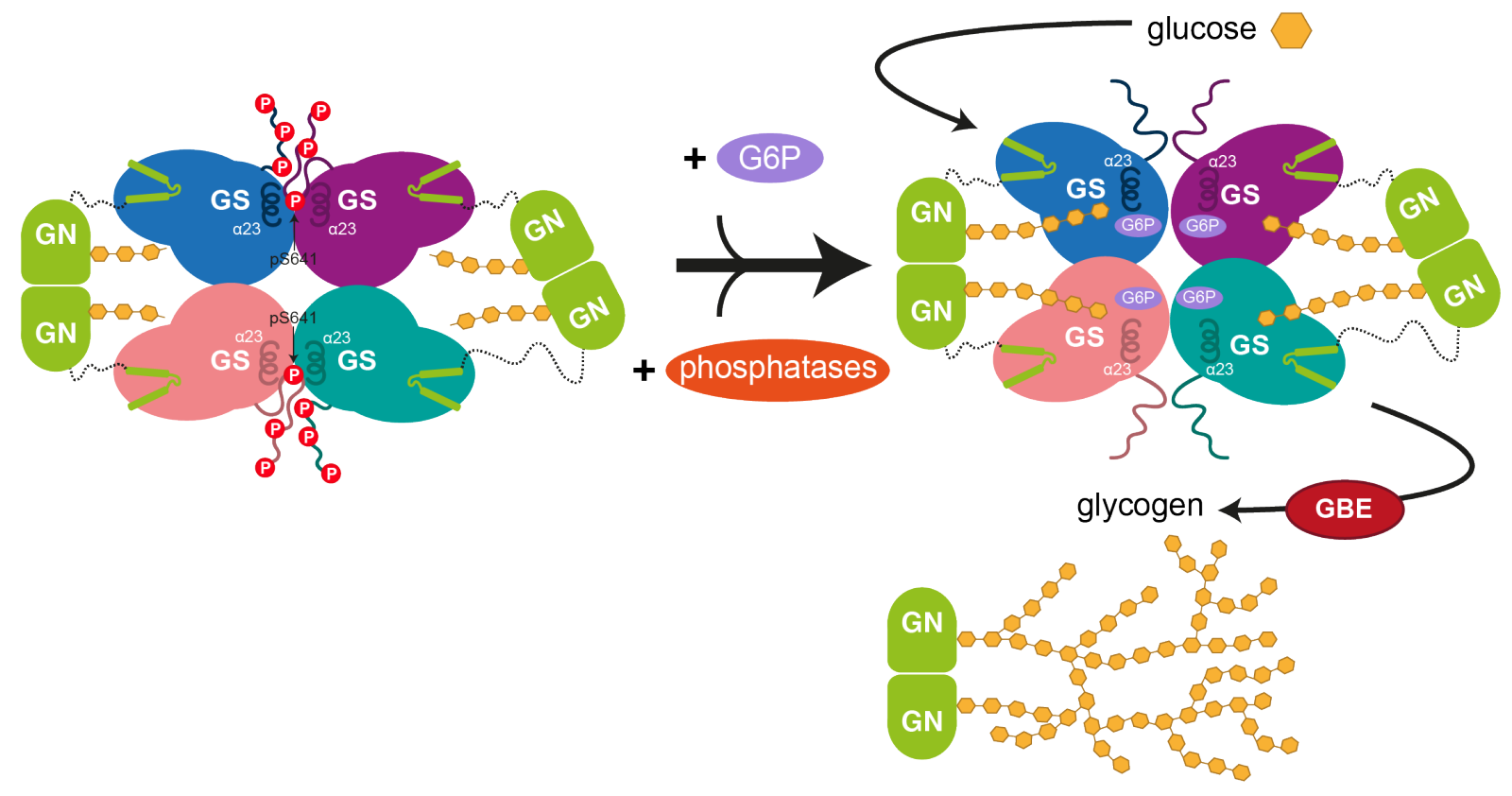

Fig. 5 GS and GN cooperate to synthesize glycogen

Glucose is converted into glycogen through the action of glycogenin (GN), glycogen synthase (GS) and glycogen branching enzyme (GBE). GN interacts with GS to feed the initial glucose chain into the GS active site for elongation. GS is regulated by allosteric activation and inhibitory phosphorylation. pS641 from one C- terminal tail interacts with the regulatory helices $\alpha 23$.

414 Dephosphorylation of the GS-GN(Y195F) complex resulted in an increase in basal activity, 415 yet there is little difference in the high activity (G6P-bound) state between phosphorylated and 416 dephosphorylated complexes (Fig. 3a and Fig. 4d). This is in accordance with previous 417 studies that demonstrate that G6P can overcome inhibition by phosphorylation and restore full 418 activity $^{2}$. C-terminal mutants R588A+R591A and R603A and the N-terminal W18A mutant 419 were more easily dephosphorylated than WT (Fig. 4c) suggesting that dislodging of the phosphoregulatory region leads to phosphorylation sites being more accessible to phosphatases.

423 The robust dephosphorylation of GS R588A+R591A did not result in an increase in basal 424 activity (Fig. 4c and 4d), which mirrors previous results in yeast ${ }^{30}$. The analogous mutations 425 were used in yGS resulting in low basal activity, yet it could still be fully activated by $\mathrm{G}^{30}$. It 426 was proposed that these residues in the regulatory helix are essential for keeping GS in a 427 "spring loaded" intermediate state, and thus charge neutralization by mutation of arginine to alanine leads to the "tense" inactive state ${ }^{30}$. Our activity data agree with this as we don't see an increase in basal activity despite dephosphorylation at S641 and S8, although we do see a marginal difference between this mutant and WT in the phosphorylated state (Fig. 4a and 
431 4c). However, it is important to note that we also see some complex dissociation with the

432 R588A+R591A mutant as evidenced by a larger dissociated complex peak in mass

433 photometry (Supplementary Fig. 9c). It is possible that the dephosphorylated R588A+R591A

434 mutant is also unstable and even more dissociated than the phosphorylated mutant, resulting

435 in a less active preparation.

Mutations of human GS1 and GS2 are prevalent in glycogen storage diseases. Several mutations cause glycogen storage disease 0 , which is due to a deficiency in GS and thus reduced levels of glycogen ${ }^{43}$. These mutations are enriched in key binding pockets of GS, affecting UDP-G, G6P and sugar binding. Some mutations affect the interaction between GS and $\mathrm{GN}^{34}$, consistent with the requirement of this interaction for glycogen synthesis ${ }^{19}$. Thus, the structure provides a valuable resource to understand disease mutations and to assess the impact residue changes have on GS-GN activity and/or interaction (Supplementary Table 2 and Supplementary Fig. 8f).

Our phosphorylated GS-GN structure reveals that the $\mathrm{N}$ - and C-terminal phosphorylation tails constrain inter-subunit movement by coordinating the $\mathrm{N}$-terminal lobes of GS and interaction of the regulatory helices $\alpha 23$ primarily via an arginine-rich pocket (arginine cradle). Thus, GS has evolved a mechanism by which the phosphorylated $\mathrm{N}$ - and $\mathrm{C}$-terminal "spike" regions hold GS in an inactive conformation that is relieved by dephosphorylation and/or G6P binding. We propose that the dynamic nature of these "spike" regions serves the purpose of exposing phosphorylated residues to phosphatases, thus allowing a "tuneable rheostat" instead of an on/off switch for regulating GS activity. Collectively, our analyses of the human GS-GN enzyme complexes reveal important mechanistic and structural details that could improve our understanding of glycogen storage diseases.

\section{Materials and Methods}

\section{Materials}

461 Total GN antibody (S197C, third bleed) was obtained from MRC-PPU Reagents and Services.

462 Total GS (\#3898) and p-GS S641 (\#47043) antibodies were from Cell Signaling Technologies. 463 p-GS S641/S645 (07-817) is from MerckMillipore. p-GS S8 antibody (YZ5716, first bleed) was 464 generated by (YenZym Antibodies Brisbane, CA, USA) by immunisation with a phosphorylated 465 peptide of the mouse GYS1 sequence (PLSRSL-*S-VSSLPG-amide, the prefix * denotes the 466 phosphorylated residue). Secondary antibodies (711-035-152 and 713-035-147) were 
obtained from BioRad. Glucose-6-phosphate (G6P) (10127647001) is from Roche. All other chemicals if not noted otherwise are from Sigma Aldrich.

\section{Cloning, protein expression and purification of GS-GN complex}

471 Genes encoding human GS1 (HsGS:NM 002103) and human GN1 (HsGN:NM 001184720)

472 mutant were cloned into $\mathrm{pFL}$ a vector ${ }^{44}$. A single $6 \mathrm{x}$ His purification tag followed by a cleavable

473 site was engineered at the N-terminus of GN WT or GN Y195F mutant. For co-expression of

474 WT GS and mutants the genes encoding human GS1 and human GN1 (Y195F) were cloned 475 in pFastBac vectors, both with a $6 \times$ His purification tag followed by a TEV site at the $\mathrm{N}$ 476 terminus. Recombinant bacmids were generated in $\mathrm{DH} 10 \mathrm{Bac}^{\mathrm{TM}}$ cells. Virus amplification and cell expression in Spodoptera frugiperda (sf9) cells and Trichoplusia ni (Tni) cells respectively were carried out using standard procedures ${ }^{45}$. For co-infection of pFastBac clones, a 10:1 ratio of the GS:GN P2 virus ratio was used. A PCR based site directed mutagenesis was used to create the following mutants from the pFastBac GS1 construct: W18A, R588A+R591A, Y600A, R603A, H610E, S614R. All of the alterations were confirmed by DNA sequencing.

Cell pellets containing HsGS-GN, HsGS-GN(Y195F) and mutants were resuspended in lysis buffer (50 mM Tris-HCl pH 7.6, $300 \mathrm{mM} \mathrm{NaCl}, 20 \mathrm{mM}$ imidazole, 10\% glycerol, 0.075\% $\beta$ mercaptoethanol, $1 \mathrm{mM}$ benzamidine, $0.8 \mathrm{mM}$ phenylmethyl sulfonyl fluoride (PMSF), 0.3 $\mathrm{mg} / \mathrm{mL}$ lysozyme). Cells were lysed by sonication ( 1 second on, 3 seconds off for a total of 5 minutes) on ice and the lysate was cleared by centrifugation at $35,000 \mathrm{~g}$ for 30 minutes at $4{ }^{\circ} \mathrm{C}$. The clarified lysate was sonicated again ( 1 second on, 3 seconds off for a total of 1 minute), followed by filtering with a $0.45 \mu \mathrm{M}$ filter (MerckMillipore). Filtered lysate was loaded onto a pre-equilibrated $1 \mathrm{~mL}$ or $5 \mathrm{~mL}$ HisTrap HP column (GE Healthcare) charged with $\mathrm{Ni}^{2+}$. The loaded column was washed with four column volumes (CV) of low salt buffer (50 mM Tris$\mathrm{HCl} \mathrm{pH} \mathrm{7.6,} 300 \mathrm{mM} \mathrm{NaCl}, 20 \mathrm{mM}$ imidazole, 10\% glycerol, $0.075 \% \beta$-mercaptoethanol, $1 \mathrm{mM}$ benzamidine), followed by four $\mathrm{CV}$ washes of high salt buffer $(50 \mathrm{mM}$ Tris- $\mathrm{HCl} \mathrm{pH} 7.6,500$ $\mathrm{mM} \mathrm{NaCl}, 20 \mathrm{mM}$ imidazole, 10\% glycerol, 0.075\% $\beta$-mercaptoethanol, $1 \mathrm{mM}$ benzamidine) and finally $4 \mathrm{CV}$ washes in low salt buffer. The column was then attached to the AKTA system (GE Healthcare) and washed with low salt buffer. The protein was then eluted by applying an imidazole gradient with elution buffer $(50 \mathrm{mM}$ Tris- $\mathrm{HCl} \mathrm{pH} 7.6,300 \mathrm{mM} \mathrm{NaCl}, 300 \mathrm{mM}$ imidazole, $10 \%$ glycerol, $0.075 \% \beta$-mercaptoethanol, $1 \mathrm{mM}$ benzamidine). The fractions containing protein were analysed by SDS-PAGE and then pooled and dialysed overnight (10,000 MWCO SnakeSkin dialysis tubing (Thermo Scientific)) at $4{ }^{\circ} \mathrm{C}$ in dialysis buffer with

501 TEV protease added (50 mM Tris- $\mathrm{HCl} \mathrm{pH} \mathrm{7.6,} 150 \mathrm{mM} \mathrm{NaCl}, 20 \mathrm{mM}$ imidazole, 10\% glycerol, $502 \quad 0.075 \% \beta$-mercaptoethanol, $1 \mathrm{mM}$ benzamidine). The dialysed protein was re-loaded onto the 
503 HisTrap column equilibrated with low salt buffer, for a Ni subtraction step. TEV cleaved protein

504 was eluted in the flow through and low salt washes. The flow through and first low salt wash

505 were pooled and concentrated using a VIVASPIN20 30,000 MWCO (Sartorius, Generon),

506 followed by centrifugation at $17,000 \mathrm{~g}$ for 15 minutes at $4{ }^{\circ} \mathrm{C}$. Protein was then injected onto a

$50716 / 600$ or 10/300 Superdex200 column (GE Healthcare) equilibrated with gel filtration buffer

508 (25 mM HEPES pH 7.5, $150 \mathrm{mM} \mathrm{NaCl}, 1 \mathrm{mM}$ TCEP, 10\% glycerol). Fractions containing

509 protein were analysed by SDS-PAGE, and fractions containing GN were pooled, concentrated

510 and stored at $-80^{\circ} \mathrm{C}$. Some fractions containing GS-GN complex were stored separately at -

$51180^{\circ} \mathrm{C}$ and the remaining protein was pooled and concentrated before being stored at $-80^{\circ} \mathrm{C}$.

512 Proteins were visualized by Coomassie blue staining, and glucosylated species were detected 513 using the periodic acid-Schiff (PAS) method (Glycoprotein staining kit, Thermo Scientific).

514

\section{In vitro dephosphorylation of GS-GN}

516 Protein phosphatase 1 (PP1) and lambda phosphatase (lambda PP) were bought from MRC 517 PPU Reagents and Services. Both have an N-terminal GST tag and lambda PP also has a C518 terminal 6xHis tag. GS-GN complex was dephosphorylated in reactions containing equal 519 amounts of PP1 and lambda PP in 25 mM HEPES pH 7.5, $150 \mathrm{mM} \mathrm{NaCl}, 1 \mathrm{mM}$ TCEP, $1 \mathrm{mM}$ $520 \mathrm{MnCl}_{2}$ and $10 \%$ glycerol for 30 minutes at $30{ }^{\circ} \mathrm{C}$. For subsequent differential scanning 521 fluorimetry experiments, the phosphatases were removed by incubating the reactions with 522 GST beads for 1 hour at $4{ }^{\circ} \mathrm{C}$. Reactions were then passed through an equilibrated $0.45 \mu \mathrm{m}$ 523 Spin-X column (Costar, $0.45 \mu \mathrm{m}$ cellulose acetate) and eluted by centrifugation at $16,000 \mathrm{~g}$ for 5242 minutes.

525

526 Negative stain electron microscopy - grid preparation and data collection

527 HsGS-GN WT and Y195F were diluted in buffer (25 mM HEPES pH 7.5, $150 \mathrm{mM} \mathrm{NaCl}, 1 \mathrm{mM}$ 528 TCEP, $10 \%$ glycerol) to concentrations between 0.01 and $0.02 \mathrm{mg} / \mathrm{mL}$ immediately before grid 529 preparation. Carbon-coated copper grids (Formvar/Carbon 300 mesh Cu, Agar Scientific) 530 were glow-discharged for 30 seconds, $10 \mathrm{~mA}$ and $0.39 \mathrm{mBar}$ pressure (PELCO easiGlow, Ted 531 Pella). Grids were incubated for 1 minute with $6 \mu \mathrm{L}$ sample, washed with $\mathrm{H}_{2} \mathrm{O}$ three times and 532 stained twice with $2 \% \mathrm{w} / \mathrm{v}$ uranyl acetate for 20 seconds. Excess liquid was removed by 533 blotting with filter paper. Data was collected on a FEI Technai F20 electron microscope 534 operated at $120 \mathrm{keV}$, equipped with a FEI Ceta (CMOS CCD) camera.

\section{Negative stain electron microscopy - data processing}

537 RELION 3.0 was used for processing of negative stain-EM data ${ }^{46}$. Real-time contrast transfer 538 function (CTF) parameters were determined using gCTF $^{47}$. Approximately 2,000 particles 
539 were manually picked, extracted with a box size of $104 \AA^{2}$, then subjected to reference-free

$5402 \mathrm{D}$ classification to produce initial references to be used for auto-picking. The parameters for

541 auto-picking were optimized and 92,580 particles were extracted. The extracted particles were

542 used for iterative rounds of reference-free 2D classification. Based on visual inspection, best

543 quality 2D average classes were selected to generate a de novo 3D initial model, which was

544 used as a reference in unsupervised 3D classification. These classes were then subjected to

$5453 \mathrm{D}$ refinement to generate a final EM density map.

546

547 Cryo-electron microscopy - grid preparation and data collection

548 Quantifoil R2/1 Cu300 or Quantifoil R1.2/1.3 Cu300 (Quantifoil Micro Tools) grids were glow549 discharged using the GloQube plasma cleaner (Quorum) at $40 \mathrm{~mA}$ for 30 seconds, for GS$550 \mathrm{GN}(\mathrm{Y} 195 \mathrm{~F})$ and GS-GN respectively. A FEI Vitrobot IV was equilibrated at $4{ }^{\circ} \mathrm{C}$ at $100 \%$ 551 relative humidity. GS-GN(Y195F) complex was diluted in buffer (25 mM HEPES pH7.5, 150 $552 \mathrm{mM} \mathrm{NaCl}, 1 \mathrm{mM}$ TCEP) to $0.23 \mathrm{mg} / \mathrm{mL}(1.85 \mu \mathrm{M})$ containing $3 \%$ glycerol immediately before

$5533 \mu \mathrm{L}$ was added to the grid. GS-GN was diluted to $0.36 \mathrm{mg} / \mathrm{mL}(2.97 \mu \mathrm{M})$ containing $8 \%$ 554 glycerol. This was followed by immediate blotting and plunge-freezing into liquid ethane cooled 555 by liquid nitrogen.

556

557 All data was collected on a FEI Titan KRIOS transmission electron microscope at $300 \mathrm{keV}$.

558 The total electron dose was $85 \mathrm{e} / \AA^{2}$, a magnification of $75,000 \mathrm{x}$ was used and a final calibrated 559 object sampling of $1.065 \AA /$ pixel. For GS-GN(Y195F), 8,699 movies were recorded using the 560 EPU automated acquisition software on a FEI Falcon III direct electron detector in integrating 561 mode $^{48}$. Each movie had a total exposure time of 1.8 seconds, collected over 47 fractions with 562 an electron dose of $1.8 \mathrm{e} / \AA^{2}$ per fraction. Three exposures per hole was taken and the defocus 563 values ranged from $-1.7 \mu \mathrm{m}$ to $-3.1 \mu \mathrm{m}$.

565 For GS-GN, 3,009 movies were recorded using the EPU automated acquisition software on a 566 FEI Falcon III direct electron detector in integrating mode ${ }^{48}$. Each movie had a total exposure 567 time of 1.6 seconds, collected over 47 fractions with an electron dose of $1.8 \mathrm{e} / \AA^{2}$ per fraction. 568 One exposure per hole was taken and the defocus values ranged from $-1.7 \mu \mathrm{m}$ to $-3.1 \mu \mathrm{m}$.

\section{Cryo-electron microscopy - data processing}

571 For both GS-GN(Y195F) and GS-GN, drift-corrected averages of each movie were created 572 using MotionCor $2^{49}$ and real-time contrast transfer function (CTF) parameters were 573 determined using $\mathrm{gCTF}^{47}$. Both motion correction and CTF estimation were carried out on-the $574 \mathrm{fly}^{48}$. Particles were picked using the PhosaurusNet general model in crYOLO v1.3.5 $5^{50}$. For 
575 GS-GN(Y195F), 780,290 particles were picked and for GS-GN, 250,250 particles were picked.

576 Particles were then imported into RELION 3.046, extracted with a box size of 220 pixels and

577 subjected to reference-free 2D classification.

578

579 For GS-GN(Y195F), 546,661 particles selected after 2D classification were subjected to 3D 580 classification, applying D2 symmetry. 3D classification gave a multitude of conformations, 581 indicating flexibility and heterogeneity in the dataset. To treat this heterogeneity, iterative 582 rounds of 3D classification were performed, generating 12 classes each round. Carrying all 583 "good"/unambiguous classes forward, 160,687 particles were subjected to 3D refinement and 584 postprocessing, generating a map at $4.11 \AA$. Followed by iterative rounds of per particle 585 contrast transfer function refinement and Bayesian particle polishing to generate a map at 4 586 A. Final resolutions were determined using the gold-standard Fourier shell correlation criterion 587 (FSC=0.143). Local resolution was estimated using the local resolution feature in RELION.

588

589 To prevent interpretation of any artefacts created by applying D2 symmetry, the data was also 590 processed in C1 symmetry. The same particles after 2D classification were subjected to 3D 591 classification without applying symmetry. All "good"/unambiguous classes were carried 592 forward for a second round of 3D classification. High quality classes were then subjected to $5933 \mathrm{D}$ refinement and postprocessing, to generate a $4.8 \AA$ map.

594

595 To elucidate the movement of phosphoregulatory regions, an alignment free 3D classification 596 with a mask containing the "spike" density was performed, using a regularisation parameter T 597 of $40^{51}$ (Fig. 2c and Supplementary Fig. 4f).

599 For GS-GN, 84,557 particles selected after 2D classification were subjected to 3D 600 classification. 36,972 particles (from 2 classes) were subjected to $3 \mathrm{D}$ refinement and 601 postprocessing, generating a map at $6.0 \AA$.

602

603 To explore the heterogeneity in the dataset, the 3D variability analysis ${ }^{35}$ tool in cryoSPARC 604 v3.2.0 $0^{36}$ was used. The 160,687 particles after 3D classification were imported into 605 cryoSPARC and homogenous refinement with a mask was performed. The subsequent 606 particles were used in the 3D variability analysis to solve 3 modes, using $C 1$ symmetry and 607 with a filter resolution of $7 \AA$. The subsequent frames were visualized in Chimera ${ }^{52} .1$ mode is 608 shown in Movie S1. 


\section{Model building and refinement}

611 A preliminary model of human GS was generated by AlphaFold ${ }^{40}$. This preliminary model was

612 rigid body fitted into the cryoEM density in UCSF Chimera ${ }^{52}$. The model was then built using

613 iterative rounds of manual building in $\mathrm{COOT}^{53}$ and real space refinement in PHENIX $1.14^{54}$. A

614 model of the last 34 residues of human GN was created by $P$ hyre $2^{55}$ and placed in the 615 approximate location using the C. elegans GS-GN ${ }^{34}$ crystal structure (PDB ID 4QLB) in 616 Chimera $^{52}$ and then fitted into the map by rigid body fitting.

617

618 Visualisation, structure analysis and sequence alignments

619 Visualisation and structure analysis were performed using ChimeraX ${ }^{56}$ or Chimera ${ }^{52}$. Multiple 620 sequence alignments were performed using MUSCLE ${ }^{57}$ and displayed and edited using 621 ALINE v1.0.025

622

\section{Mass photometry}

624 Mass photometry experiments were performed using a Refyn One ${ }^{\mathrm{MP}}$ mass photometer. 625 Immediately prior to mass photometry measurements, proteins were diluted in 25 mM HEPES

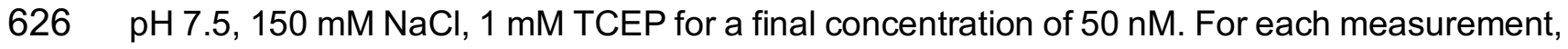
627 (16 $\mu \mathrm{L})$ buffer was added to a well and the focus point was found and adjusted when 628 necessary. Protein $(4 \mu \mathrm{L})$ was then added to the buffer droplet, the sample was mixed and movies of 60 seconds were recorded using AcquireMP. Data were analysed using

630 DiscoverMP.

631

\section{Differential Scanning Fluorimetry}

633 Thermal shift assays were performed using an Applied Biosystems QuantStudio 3 Real-Time 634 PCR system. SYPRO ${ }^{\text {TM }}$ Orange (Invitrogen) was used as a fluorescence probe. Proteins were 635 diluted in $25 \mathrm{mM}$ HEPES pH 7.5, $150 \mathrm{mM} \mathrm{NaCl}, 1 \mathrm{mM}$ TCEP to a final concentration of $1 \mu \mathrm{M}$. 636 Varied concentrations of G6P were added and the reaction was incubated at room 637 temperature for 30 minutes. SYPRO Orange was diluted in $25 \mathrm{mM} \mathrm{HEPES} \mathrm{pH} \mathrm{7.5,} 150 \mathrm{mM}$ $638 \mathrm{NaCl}, 1 \mathrm{mM}$ TCEP to a final concentration of $2.5 \mathrm{X}$, in a total reaction volume of $20 \mu \mathrm{L}$. The 639 temperature was raised in $0.018^{\circ} \mathrm{C}$ intervals from $20^{\circ} \mathrm{C}$ to $95^{\circ} \mathrm{C}$. Data were analysed using 640 Protein Thermal Shift v1.4.

\section{Tandem mass spectrometry}

643 Concentrated purified protein complexes $(6.75 \mu \mathrm{g})$ were diluted 30 -fold in $25 \mathrm{mM}$ ammonium 644 bicarbonate $\mathrm{pH} 8.0$ before being subject to reduction with dithiothreitol and alkylation with 645 iodoacetamide, as previously described ${ }^{59}$. The eluent was equally divided into three for 
digestion with either: 33:1 (w/w) trypsin gold (Promega), 25:1 (w/w) chymotrypsin (Promega), or 10:1 (w/w) elastase (Promega), using the manufacturer's recommended temperatures for 18 hours with $600 \mathrm{rpm}$ shaking. Digests were then subject to in-house packed, strong cation exchange stage tip clean-up, as previously described by ${ }^{60}$. Dried peptides were solubilized in $20 \mu \mathrm{l}$ of $3 \%(\mathrm{v} / \mathrm{v})$ acetonitrile and $0.1 \%(\mathrm{v} / \mathrm{v})$ TFA in water, sonicated for 10 minutes, and centrifuged at $13,000 \mathrm{~g}$ for 15 minutes at $4{ }^{\circ} \mathrm{C}$ being separated using an Ultimate 3000 nano system (Dionex) by reversed-phase HPLC, over a 60-minute gradient, as described in ${ }^{59}$. All data acquisition was performed using a Thermo Orbitrap Fusion Lumos Tribrid mass spectrometer (Thermo Scientific), with higher-energy C-trap dissociation (HCD) fragmentation set at $32 \%$ normalized collision energy for $2+$ to $5+$ charge states. MS1 spectra were acquired in the Orbitrap $(60 \mathrm{~K}$ resolution at $200 \mathrm{~m} / \mathrm{z})$ over a range of 350 to $1400 \mathrm{~m} / \mathrm{z}$, AGC target = standard, maximum injection time $=$ auto, with an intensity threshold for fragmentation of $2 \mathrm{e}^{4}$. MS2 spectra were acquired in the Orbitrap (30K resolution at $200 \mathrm{~m} / \mathrm{z}$ ), AGC target = standard, maximum injection time $=$ dynamic. A dynamic exclusion window of 20 seconds was applied at a $10 \mathrm{ppm}$ mass tolerance. Data was analysed by Proteome Discoverer 1.4 using the UniProt Human reviewed database (updated April 2020) with fixed modification = carbamidomethylation $(C)$, variable modifications $=$ oxidation $(M)$ and phospho $(S / T / Y)$, instrument type = electrospray ionization-Fourier-transform ion cyclotron resonance (ESIFTICR), MS1 mass tolerance $=10 \mathrm{ppm}, \mathrm{MS} 2$ mass tolerance $=0.01 \mathrm{Da}$, and the ptmRS node on; set to a score $>99.0$.

\section{Glycogen synthase activity assay}

$6681 \mu \mathrm{g}$ of purified protein was diluted in ice cold lysis buffer $(270 \mathrm{mM}$ sucrose, $50 \mathrm{mM}$ Tris $\mathrm{HCl}$ (pH 7.5), 1 mM EDTA, 1 mM EGTA, 1\% (v/v) Triton X-100, 20 mM glycerol-2-phosphate, 50 $\mathrm{mM} \mathrm{NaF}, 5 \mathrm{mM} \mathrm{Na} \mathrm{P}_{2} \mathrm{O}_{7}, 1 \mathrm{mM}$ DTT, $0.1 \mathrm{mM}$ PMSF, $1 \mathrm{mM}$ benzamidine, $1 \mathrm{mg} / \mathrm{mL}$ microcystin-LR, $2 \mathrm{mg} / \mathrm{mL}$ leupeptin, and $2 \mathrm{mg} / \mathrm{mL}$ pepstatin A) to a total volume of $100 \mu \mathrm{L} .20$

$672 \mu \mathrm{L}$ of the protein was added to $80 \mu \mathrm{L}$ of the assay buffer $(25 \mathrm{mM}$ Tris-HCl $(\mathrm{pH} 7.8), 50 \mathrm{mM}$

$673 \mathrm{NaF}, 5 \mathrm{mM}$ EDTA, $10 \mathrm{mg} / \mathrm{ml}$ glycogen, $1.5 \mathrm{mM}$ UDP-glucose, 0.125\% (v/v) 2-mercaptoethanol 674 and $0.15 \mathrm{mCi} / \mathrm{mmol} \mathrm{D}-\left[{ }^{14} \mathrm{C}\right]-U D P-g l u c o s e$ (American Radiolabelled Chemicals, Inc., ARC 675 0154) with 0 and $12.5 \mathrm{mM} \mathrm{G6P}$. Reactions were incubated for 20 minutes at $30^{\circ} \mathrm{C}$ with mild agitation at $300 \mathrm{rpm}$. The reactions were stopped by spotting $90 \mu \mathrm{L}$ of the reaction mix onto $2.5 \mathrm{~cm} \times 2.5 \mathrm{~cm}$ squares of filter paper (Whatman 3MM) which were immediately immersed in ice cold $66 \%$ ethanol and left to incubate with mild agitation for 20 minutes. The filter papers were washed thrice more in $66 \%$ ethanol for 20 minutes each and rinsed in acetone. The dried filters were subjected to scintillation counting. 


\section{Immunoblotting}

683 Purified proteins were denatured in Laemmli buffer at $100^{\circ} \mathrm{C}$ for 5 minutes. $100 \mathrm{ng}$ of the 684 protein was separated by SDS-PAGE on $4-10 \%$ gel and transferred onto nitrocellulose membranes (\#926-31090, LiCOR). Membranes were blocked for 45 minutes at room temperature in 5\% skim milk (Sigma, 1153630500) followed by incubation in TBST (10 mM Tris ( $\mathrm{pH} 7.6), 137 \mathrm{mM} \mathrm{NaCl}$, and $0.1 \%(\mathrm{v} / \mathrm{v})$ Tween-20) containing 5\% (w/v) BSA and the primary antibody overnight at $4{ }^{\circ} \mathrm{C}$. The membranes were incubated for 45 minutes in HRP conjugated secondary antibodies diluted 1:10,000 in 3\% skim milk, after extensive washing in TBST. The membranes were imaged using enhanced chemiluminescence $(E C L)$ reagent (GE Healthcare).

\section{Author contributions:}

694 L.M. performed the majority of experiments (molecular biology, protein production, electron 695 microscopy, differential scanning fluorimetry and mass photometry analysis), D.B. performed 696 glycogen synthase activity and western blot assays, L.D. performed phosphorylation mapping 697 and C.B. provided support with structural biology and protein production. L.M. and E.Z. drafted 698 the manuscript with input from D.B., K.S., L.D. and C.E.E. and all authors revised it. J.P., 699 C.E.E., C.H., J.B., N.A.R., H.K., K.S. and E.Z. provided supervision and project management. 700 E.Z., K.S., C.E.E., C.H. and J.B. designed and interpreted data in consultation with all authors.

\section{Acknowledgments:}

702 L.M. is supported by an MRC Discovery Medicine North (DiMeN) iCASE studentship co703 funded by UKRI and Vertex Pharmaceuticals. D.B. is supported by an International 704 Postdoctoral Fellowship funded by the Novo Nordisk Foundation Center for Basic Metabolic 705 Research. C.E. and L.D. were supported by grants from BBSRC (BB/S018514/1, 706 BB/M012557/1, BB/R000182/1). E.Z. is supported by a Sir Henry Dale Fellowship from the 707 Wellcome Trust \& Royal Society (200523/Z/16/Z). The Astbury cryo-EM Facility is funded by 708 a University of Leeds ABSL award and a Wellcome Trust (108466/Z/15/Z) grant. The Novo 709 Nordisk Foundation Center for Basic Metabolic Research is an independent Research Center 710 based at the University of Copenhagen, Denmark, and partially funded by an unconditional 711 donation from the Novo Nordisk Foundation (Grant number NNF18CC0034900).

\section{Conflict of Interest}

714 The authors report no conflicts of interest. 


\section{References}

718 1. Roach, P. J., Depaoli-Roach, A. A., Hurley, T. D. \& Tagliabracci, V. S. Glycogen and its metabolism: some new developments and old themes. Biochem. J. 441, 763-787

721 (2012).

722

2. Roach, P. J. Glycogen and its metabolism. Curr. Mol. Med. 2, 101-120 (2002).

723

3. Pitcher, J., Smythe, C., Campbell, D. C

G. \& Cohen, P. Identification of the 38-kDa

724 subunit of rabbit skeletal muscle glycogen synthase as glycogenin. European Journal of Biochemistry 169, 497-502 (1987).

726

4. Zeqiraj, E. \& Sicheri, F. Getting a handle on glycogen synthase - Its interaction with glycogenin. Molecular Aspects of Medicine 46, 63-69 (2015).

728

5. Skurat, A. V., Dietrich, A. D. \& Roach, P. J. Interaction between glycogenin and

729 glycogen synthase. Archives of Biochemistry and Biophysics 456, 93-97 (2006).

730

731

732

7. Ryu, J.-H. et al. Comparative structural analyses of purified glycogen particles from

6. Pitcher, J., of, C. S. E. J.1988. Glycogenin is the priming glucosyltransferase required for the initiation of glycogen biogenesis in rabbit skeletal muscle. European Journal of Biochemistry 176, 391-395 (1988).

738

739 rat liver, human skeletal muscle and commercial preparations. International Journal of Biological Macromolecules 45, 478-482 (2009).

8. Ashcroft, F. M., Rohm, M., Clark, A. \& Brereton, M. F. Is Type 2 Diabetes a Glycogen

11. Malfatti, E. et al. A new muscle glycogen storage disease associated with glycogenin1 deficiency. Ann Neurol. 76, 891-898 (2014).

12. Pursell, N. et al. Inhibition of Glycogen Synthase II with RNAi Prevents Liver Injury in Mouse Models of Glycogen Storage Diseases. Mol Ther 26, 1771-1782 (2018).

745 13. Ashe, K. M. et al. Inhibition of glycogen biosynthesis via mTORC1 suppression as an adjunct therapy for Pompe disease. Molecular Genetics and Metabolism 100, 309315 (2010).

748 14. Mu, J., Skurat, A. V. \& Roach, P. J. Glycogenin-2, a Novel Self-glucosylating Protein Involved in Liver Glycogen Biosynthesis. J. Biol. Chem. 272, 27589-27597 (1997). 
15. Curtino, J. A. \& Aon, M. A. From the seminal discovery of proteoglycogen and glycogenin to emerging knowledge and research on glycogen biology. Biochem. J. 476, 3109-3124 (2019).

16. Lairson, L. L., Henrissat, B., Davies, G. J. \& Withers, S. G. Glycosyltransferases: Structures, Functions, and Mechanisms. Annu. Rev. Biochem. 77, 521-555 (2008).

17. Gibbons, B. J., Roach, P. J. \& Hurley, T. D. Crystal Structure of the Autocatalytic Initiator of Glycogen Biosynthesis, Glycogenin. Journal of Molecular Biology 319, 463-477 (2002).

18. Chaikuad, A. et al. Conformational plasticity of glycogenin and its maltosaccharide substrate during glycogen biogenesis. Proceedings of the National Academy of Sciences 108, 21028-21033 (2011).

19. Zeqiraj, E. et al. Structural basis for the recruitment of glycogen synthase by glycogenin. Proceedings of the National Academy of Sciences 111, E2831-E2840 (2014).

20. Skurat, A. V., Wang, Y. \& Roach, P. J. Rabbit skeletal muscle glycogen synthase expressed in COS cells. Identification of regulatory phosphorylation sites. J. Biol. Chem. 269, 25534-25542 (1994).

21. Wilamowitz-Moellendorff, von, A. et al. Glucose-6-phosphate-mediated activation of liver glycogen synthase plays a key role in hepatic glycogen synthesis. Diabetes $\mathbf{6 2}$, 4070-4082 (2013).

22. Browner, M. F., Nakano, K., Bang, A. G. \& Fletterick, R. J. Human muscle glycogen synthase cDNA sequence: a negatively charged protein with an asymmetric charge distribution. Proceedings of the National Academy of Sciences 86, 1443-1447 (1989).

23. Nuttall, F. Q., Gannon, M. C., Bai, G. \& Lee, E. Y. Primary structure of human liver glycogen synthase deduced by cDNA cloning. Archives of Biochemistry and Biophysics 311, 443-449 (1994).

24. Buschiazzo, A. et al. Crystal structure of glycogen synthase: homologous enzymes catalyze glycogen synthesis and degradation. The EMBO Journal 23, 3196-3205 (2004).

25. Skurat, A. V., Peng, H. L., Chang, H. Y., Cannon, J. F. \& Roach, P. J. Ratedetermining steps in the biosynthesis of glycogen in COS cells. Archives of Biochemistry and Biophysics 328, 283-288 (1996).

26. Hunter, R. W., Treebak, J. T., Wojtaszewski, J. F. P. \& Sakamoto, K. Molecular Mechanism by Which AMP-Activated Protein Kinase Activation Promotes Glycogen Accumulation in Muscle. Diabetes 60, 766-774 (2011). 
790

791

792

793

794

795

796

797

798

799

800

801

802

803

804

805

806

807

808

809

810

811

812

813

814

815

816

817

818

819

820

27. Baskaran, S., Roach, P. J., Depaoli-Roach, A. A. \& Hurley, T. D. Structural basis for glucose-6-phosphate activation of glycogen synthase. Proc. Natl. Acad. Sci. U.S.A. 107, 17563-17568 (2010).

28. Roach, P. J. Multisite and hierarchal protein phosphorylation. J. Biol. Chem. 266, 14139-14142 (1991).

29. Roach, P. J. Control of glycogen synthase by hierarchal protein phosphorylation. FASEB J 4, 2961-2968 (1990).

30. Mahalingan, K. K., Baskaran, S., Depaoli-Roach, A. A., Roach, P. J. \& Hurley, T. D. Redox Switch for the Inhibited State of Yeast Glycogen Synthase Mimics Regulation by Phosphorylation. Biochemistry 56, 179-188 (2017).

31. Hunter, R. W., Zeqiraj, E., Morrice, N., Sicheri, F. \& Sakamoto, K. Expression and purification of functional human glycogen synthase-1:glycogenin-1 complex in insect cells. PROTEIN EXPRESSION AND PURIFICATION 108, 23-29 (2015).

32. Khanna, M. et al. Expression and purification of functional human glycogen synthase1 (hGYS1) in insect cells. PROTEIN EXPRESSION AND PURIFICATION 90, 78-83 (2013).

33. Smythe, C., Caudwell, F. B., Ferguson, M. \& Cohen, P. Isolation and structural analysis of a peptide containing the novel tyrosyl-glucose linkage in glycogenin. The EMBO Journal 7, 2681-2686 (1988).

34. Rodriguez, I. R. \& Whelan, W. J. A novel glycosyl-amino acid linkage: rabbit-muscle glycogen is covalently linked to a protein via tyrosine. Biochemical and Biophysical Research Communications 132, 829-836 (1985).

35. Punjani, A. \& Fleet, D. J. 3D variability analysis: Resolving continuous flexibility and discrete heterogeneity from single particle cryo-EM. Joumal of Structural Biology 213, 107702 (2021).

36. Punjani, A., Rubinstein, J. L., Fleet, D. J. \& Brubaker, M. A. cryoSPARC: algorithms for rapid unsupervised cryo-EM structure determination. Nat Methods 14, 290-296 (2017).

37. Bouskila, M. et al. Allosteric Regulation of Glycogen Synthase Controls Glycogen Synthesis in Muscle. Cell Metabolism 12, 456-466 (2010).

38. McManus, E. J. et al. Role that phosphorylation of GSK3 plays in insulin and Wnt signalling defined by knockin analysis. The EMBO Journal 24, 1571-1583 (2005).

39. Hornbeck, P. V. et al. PhosphoSitePlus, 2014: mutations, PTMs and recalibrations. Nucleic Acids Res 43, D512-20 (2015).

40. Jumper, J. et al. Highly accurate protein structure prediction with AlphaFold. Nature 596, 583-589 (2021). 
41. Baskaran, S. et al. Multiple glycogen-binding sites in eukaryotic glycogen synthase are required for high catalytic efficiency toward glycogen. J. Biol. Chem. 286, 3399934006 (2011).

42. Chikwana, V. M. et al. Structural basis for 2'-phosphate incorporation into glycogen by glycogen synthase. Proc. Natl. Acad. Sci. U.S.A. 110, 20976-20981 (2013).

43. Ellingwood, S. S. \& Cheng, A. Biochemical and clinical aspects of glycogen storage diseases. Journal of Endocrinology 238, R131-R141 (2018).

44. Fitzgerald, D. J. et al. Protein complex expression by using multigene baculoviral vectors. Nat Methods 3, 1021-1032 (2006).

45. Zeqiraj, E. et al. Higher-Order Assembly of BRCC36-KIAA0157 Is Required for DUB Activity and Biological Function. Mol Cell 59, 970-983 (2015).

46. Zivanov, J. et al. New tools for automated high-resolution cryo-EM structure determination in RELION-3. eLIFE 7, (2018).

47. Zhang, K. Gctf: Real-time CTF determination and correction. Journal of Structural Biology 193, 1-12 (2016).

48. Thompson, R. F., ladanza, M. G., Hesketh, E. L., Rawson, S. \& Ranson, N. A. Collection, pre-processing and on-the-fly analysis of data for high-resolution, singleparticle cryo-electron microscopy. Nat Protoc 14, 100-118 (2018).

49. Zheng, S. Q. et al. MotionCor2: anisotropic correction of beam-induced motion for improved cryo-electron microscopy. Nat Methods 14, 331-332 (2017).

50. Wagner, T. et al. SPHIRE-crYOLO is a fast and accurate fully automated particle picker for cryo-EM. Commun Biol 2, 218-13 (2019).

51. Scheres, S. H. W. in Cryo-EM, Part B: 3-D Reconstruction 482, 295-320 (Elsevier, 2010).

52. Pettersen, E. F. et al. UCSF Chimera--a visualization system for exploratory research and analysis. J Comput Chem 25, 1605-1612 (2004).

53. Emsley, P., Lohkamp, B., Scott, W. G. \& Cowtan, K. Features and development of

54. Adams, P. D. et al. PHENIX: a comprehensive Python-based system for macromolecular structure solution. Acta Crystallogr D Biol Crystallogr 66, 213-221 (2010). web portal for protein modeling, prediction and analysis. Nat Protoc 10, 845-858 (2015).

56. Pettersen, E. F. et al. UCSF ChimeraX: Structure visualization for researchers, educators, and developers. Protein Sci 30, 70-82 (2021). 
857 57. Madeira, F. et al. The EMBL-EBI search and sequence analysis tools APIs in 2019. Nucleic Acids Res 47, W636-W641 (2019).

859 58. Bond, C. S. \& Schüttelkopf, A. W. ALINE: a WYSIWYG protein-sequence alignment editor for publication-quality alignments. Acta Crystallogr D Biol Crystallogr 65, 510512 (2009).

59. Ferries, S. et al. Evaluation of Parameters for Confident Phosphorylation Site Localization Using an Orbitrap Fusion Tribrid Mass Spectrometer. J Proteome Res 16, 3448-3459 (2017).

865

60. Daly, L. A. et al. Oxygen-dependent changes in binding partners and posttranslational modifications regulate the abundance and activity of HIF-1 $\alpha / 2 \alpha$. Sci Signal 14, (2021).

868 